\title{
Methodology to analyze attitude stability of satellites subjected to aerodynamic torques
}

\author{
David Mostaza-Prieto ${ }^{1}$ and Peter C. E. Roberts ${ }^{2}$ \\ The University of Manchester, Manchester, EnglandM139PL, UnitedKingdom.
}

\begin{abstract}
A method to analyze the stability of satellites flying in low Earth orbits is presented. Starting from the classical rotational equations of motion including gravity gradient, the influence of aerodynamic torques is studied both in the pitch and roll-yaw axes. Using concepts used to assess aircraft stability, such as stability derivatives, several ideas and recommendations are presented. The work aims to link geometrical properties with attitude response, providing a way to design satellites that actively take into account environmental torques, either by minimizing them or by utilizing them to meet mission goals.
\end{abstract}

\footnotetext{
${ }^{1}$ Researcher, School of Mechanical, Aerospace and Civil Engineering.

${ }^{2}$ Lecturer, School of Mechanical, Aerospace and Civil Engineering.
} 


\section{Nomenclature}

$$
\begin{aligned}
& I_{x}, I_{y}, I_{z}=\text { principal moments of inertia }\left[\mathrm{kg} \cdot \mathrm{m}^{2}\right] \\
& \omega_{0} \quad=\text { angular orbital velocity in a circular orbit }[\mathrm{rad} / \mathrm{s}] \\
& \phi \quad=\text { roll angle }[\mathrm{rad}] \\
& \theta \quad=\text { pitch angle }[\mathrm{rad}] \\
& \psi \quad=\text { yaw angle }[\mathrm{rad}] \\
& M \quad=\text { disturbance torque }[N . m] \\
& V_{a} \quad=\text { velocity w.r.t wind }[\mathrm{m} / \mathrm{s}] \\
& \rho \quad=\text { density }\left[\mathrm{kg} / \mathrm{m}^{3}\right] \\
& S_{\text {ref }} \quad=\text { aerodynamic reference area }\left[m^{2}\right] \\
& L_{r e f} \quad=\text { aerodynamic reference length }[m] \\
& C_{l} \quad=\text { roll moment coefficient } \\
& C_{m} \quad=\text { pitch moment coefficient } \\
& C_{n} \quad=\text { yaw moment coefficient } \\
& \vec{C}_{F} \quad=\text { aerodynamic force coefficients in body axes } \\
& \alpha \quad=\text { angle of attack }[\mathrm{rad}] \\
& \beta \quad=\text { angle of sideslip }[\mathrm{rad}] \\
& \delta_{1}, \delta_{2}=\text { angles defining the orientation of aerodynamic velocity in orbital frame }[\mathrm{rad}] \\
& \mathrm{L} \quad=\text { vehicle length }[m] \\
& \mathrm{D}=\text { diameter of the vehicle base area }\left[m^{2}\right] \\
& x_{\operatorname{cog}}=\text { distance from the leading face to the center of mass }[m] \\
& C_{D} \quad=\text { drag coefficient } \\
& C_{m_{\alpha}} \quad=\text { stability derivative, slope of the pitch coefficient w.r.t angle of attack } \\
& T_{\text {orb }} \quad=\text { orbital period }
\end{aligned}
$$

\section{Introduction}

We present in this paper a method to analyze the effect of aerodynamic torques in the attitude motion of a satellite. The aerodynamic torque, resulting from the friction of the satellite with 
the atmosphere, is an important effect for satellites orbiting in Low Earth Orbits. Its magnitude increases with decreasing altitude and is still noticeable up to $1000 \mathrm{~km}$ [1]. Since the early days of space exploration several missions have documented the side effect of aerodynamic torques, either by a quicker spin speed decay as a result of underestimation of the torque magnitude $[2,3]$ or by the use of active control in order to correct an unexpected de-pointing [4].

Below $400 \mathrm{~km}$, the aerodynamic torque normally becomes the main disturbance effect to the satellite altitude, allowing the use of it for stabilization. The ability of a spacecraft to remain stable to a certain direction is an interesting feature. It can be used for complete passive stabilization, a simple and cheap way to achieve coarse pointing requirements, or to reduce the use and magnitude of active control solutions. Broadly used passive stabilization strategies are gravity gradient $[5,6]$ or magnetic methods [7-9], well described in many textbooks [10,11] . The concept of aerodynamic stabilization was already proposed during the early days of space exploration [12-16] . Most of the literature published during these years was captured in two good review papers: Shrivaska [17] published in 1976 and Shrivaska and Modi [18] in 1983. Apart from capturing many references, Shrivaska divides the published papers into two categories that are still applicable: studies based on simple models that attempt detailed analytical and numerical analysis, and papers that study complex systems by means of simplified equations. Despite the good number of initial publications proposing the idea, the literature regarding actual applications is rather small. A few exceptions being the stabilization of the pitch axis of the Russian satellite COSMOS-149 [19], or the more recent PAMS satellite, an aerodynamic stabilized cylinder released from the Shuttle during the 77th mission in 1996. The environment analysis and spacecraft design were described by Kumar et al in [20] and [21].

During the last 35 years, the published literature on the analysis of aerodynamic torques or their potential use for stabilization has decreased noticeably. The main reason has probably been the lack of confidence in the available models to predict aerodynamic interactions. The modeling of aerodynamic torques is a complex task, affected by many uncertainties: atmospheric density, free molecular flow interaction and thermospheric winds. As a result of the uncertainties in these quantities, the aerodynamic torque has been treated classically as a disturbance torque [1], in 
contradiction with torques produced by magnetic and gravity fields or solar radiation pressure which are more predictable in nature and have been extensively used for passive or active attitude stabilization. However, during the last couple of years, recent improvements in atmospheric models, and especially the renewed interest in cheap and small missions, such as CubeSats, have increased the number of publications proposing the possibility of partial or total aero-stabilization. Scherichev et al [22-25] have published some detailed analytical and numerical studies investigating the equilibrium positions of aerodynamic and gravity gradient torques, where the aerodynamic torque is simplified to a constant drag acting on the centre of pressure, located in a principal axis of inertia. More recent papers matching Shrivaska's second category, and focusing on spacecraft design, have also been published: the stabilization of one axis considering gravity gradient has been proposed in [26] for pitch and in [27] for yaw. Psiaki [28] presents a 3-axis stabilization design for CubeSats using magnetotorquers and feathers in a shuttlecock configuration. Some non-linear simulations of different designs of small aerostable satellites are reported in [29]. The underlining idea in all cases is the creation of a so-called restoring torque by keeping the centre of mass forward of the centre of aerodynamic pressure. One of the remaining issues is the absence of commonly accepted methods for modeling and analysis as identified by Maslova et al [30].

Our goal with this study is to follow this second category of papers with a clear spacecraft design focus. With the big development of computer simulations, there is a benefit in predicting the main behavior of complex non-linear systems with simplified equations. The idea is to provide some insight into the problem to help with the design of the spacecraft geometry before the nonlinear simulations, linking geometrical properties with attitude response. In this paper, we extend the scope of the stability analysis by relaxing the axisymmetric assumption and including gravity gradient contributions in all 3 axes. Also, useful concepts already used in traditional aircraft stability analysis are proposed, such as stability derivatives, which allow a precise analysis of stability and are compatible with a more physical modeling of aerodynamic torques. The scope of the study is the region with high Knudsen numbers for sustained flight in low orbits (typically $160 \mathrm{~km}$ upwards, $\mathrm{Kn}>100$ ), where the flow around the spacecraft can be modeled as Free Molecular or rarefied and damping terms can be neglected $[15,35]$. Then, simplified sufficient conditions for stability are 
derived both for pitch and roll-yaw axes. In order to link the geometrical design with stability and response, detailed modeling of aerodynamic torques and stability derivatives is offered for several simple and more complex geometries. Finally, the effect of non-constant density and the potential occurrence of resonant phenomena are studied.

\section{Rotational equations of motion}

We propose a similar derivation to the classic gravity gradient stability analysis that can be found in many text books and course notes, and therefore easy to follow. The idea is to understand how the aerodynamic properties modify the gravity gradient stability chart. Using the same notation of [11], the orbital frame in which the satellite is to be stabilized is defined as:

- Origin : The center of mass of the satellite.

- $Z_{R}$ : points towards the center of the earth.

- $X_{R}$ : in the plane of the orbit pointing in the direction of the inertial velocity.

- $Y_{R}$ : normal to the orbit plane and completes the right-hand side orthogonal frame.

The Euler angles are defined as the rotations about the body axes: $\phi$, roll, about $X_{B}, \theta$, pitch, about $Y_{B}$ and $\psi$, yaw, around $Z_{B}$. Then, the linearized equations of motion of a satellite traveling in a circular orbit and subjected to gravity gradient torques are [11].

$$
\begin{gathered}
I_{x} \ddot{\phi}+4 \omega_{0}^{2}\left(I_{y}-I_{x}\right) \phi-\omega_{0}\left(I_{x}-I_{y}+I_{z}\right) \dot{\psi}=T_{a x}+T_{d x} \\
I_{z} \ddot{\psi}+\omega_{0}^{2}\left(I_{y}-I_{z}\right) \psi+\omega_{0}\left(I_{x}-I_{y}+I_{z}\right) \dot{\phi}=T_{a z}+T_{d z} \\
I_{y} \ddot{\theta}+3 \omega_{0}^{2}\left(I_{x}-I_{z}\right) \theta=T_{a y}+T_{d y}
\end{gathered}
$$

Gravity gradient contributions (in $x$ and $y$ axes, there is no contribution in $z$ ) have been already included in the left side of the equations. The right side is composed by a general disturbance torque $\left(T_{d}\right)$ plus a torque due to aerodynamic forces $\left(T_{a}\right)$. 
A common approach to calculate the aerodynamic torque experienced by a body is:

$$
\left[\begin{array}{c}
T_{a x} \\
T_{a y} \\
T_{a z}
\end{array}\right]=\frac{1}{2} \rho V_{a}^{2} S_{\text {ref }} L_{r e f}\left[\begin{array}{c}
C_{l}^{G} \\
C_{m}^{G} \\
C_{n}^{G}
\end{array}\right]
$$

Where $\rho$ represents the atmospheric density, $V_{a}$ is the relative velocity of the vehicle with respect to the atmosphere, $S_{\text {ref }}$ and $L_{r e f}$ are the reference surface and length, $C_{l}^{G}, C_{m}^{G}$ and $C_{n}^{G}$ are the rolling, pitching and yawing coefficients already translated to CoG. These moment coefficients are a function of the aerodynamic angles of incidence ( $\alpha$, angle of attack and $\beta$, angle of sideslip) and the characteristics of the flow. At spacecraft altitudes, Free Molecular Flow (FMF) is a reasonable and commonly used assumption. In FMF, these coefficients are generally a function of the altitude (through both the speed ratio and the atmospheric composition), and the Gas-Surface interaction (surface properties, contamination...). For a detailed analysis of how to estimate FMF coefficients refer to Sentman [31], Schamberg [32] or Cook [33].

Although the mentioned dependencies are generally non-linear, we can restrict our analysis to a range in which the non-linearities are small, considering all dependencies to be linear (following with the small angles assumption used to derive Eqs. 1 to 3). In such a case the coefficients can be expressed as a Taylor series around a reference condition:

$$
C\left(x_{1}, x_{2}, \ldots x_{n}\right)=C\left(x_{1}, x_{2}, \ldots x_{n}\right)_{r e f}+\left(\frac{\partial C}{\partial x_{1}}\right)_{r e f} \Delta x_{1} \ldots+\left(\frac{\partial C}{\partial x_{n}}\right)_{r e f} \Delta x_{n}
$$

For a circular orbit, the altitude variation is slow with respect to attitude variations, so we can neglect it. Also, due to the characteristics of FMF, the derivatives with respect to the angular rate components (damping) are very small and negligible in effect. Thus. the aerodynamic damping terms can be removed from the analysis $[15,35]$. In FMF the time to damp to one-half the initial pitch amplitude is, at most, comparable to the satellite's orbital lifetime [15]. Damping terms increase its magnitude as altitude decreases. For lower altitude regimes, such as the ones leading to re-entry, the Free Molecular Flow assumption is no longer valid and damping would need to be included.

We can express the three coefficients as functions of aerodynamic angle variations. Using the notation $\left(\frac{\partial C_{m}}{\partial x_{1}}\right)_{r e f}=C_{m_{x_{1}}}$ the moment coefficients can be expressed at the reference point $(\alpha$, 


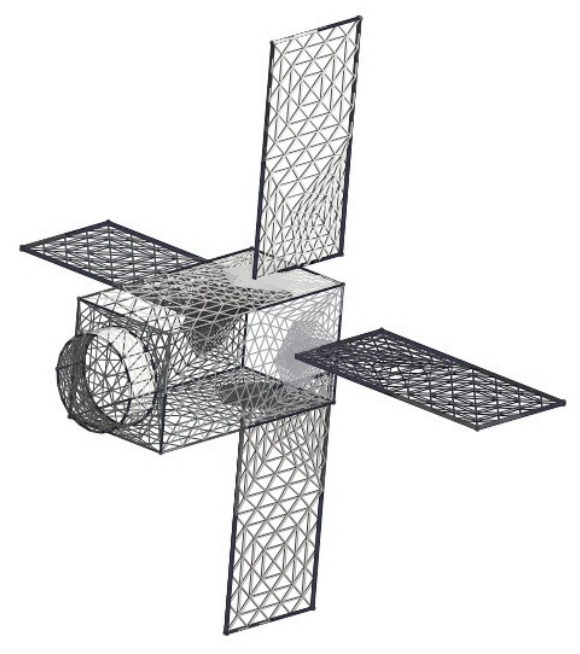

Fig. 1 Surface mesh used for FMF calculations

$\beta)=(0,0)$ as:

$$
\begin{gathered}
C_{l}=C_{l_{0}}+C_{l_{\alpha}} \alpha+C_{l_{\beta}} \beta \\
C_{m}=C_{m_{0}}+C_{m_{\alpha}} \alpha+C_{m_{\beta}} \beta \\
C_{n}=C_{n_{0}}+C_{n_{\alpha}} \alpha+C_{n_{\beta}} \beta
\end{gathered}
$$

To further simplify the problem lets assume that the spacecraft has some symmetry around the roll axis, in a way that the roll torque is negligible for any small angle of attack or sideslip $\left(C_{l}->0\right)$. This is true for many spacecraft platforms (like the one shown in Fig. 1). Also, for small angles we can consider the pitch and yaw coefficients to be dependent only on angle of attack and sideslip respectively. Assuming also no z or y CoG offsets both $C_{m_{0}}$ and $C_{n_{0}}$ are 0 , therefore, as first approach, we can consider:

$$
\begin{gathered}
C_{l}=0 \\
C_{m}=C_{m_{\alpha}} \alpha \\
C_{n}=C_{n_{\beta}} \beta
\end{gathered}
$$

The validity of the above assumptions can be seen in Fig. 2 and Fig. 3 for the FMF coefficients 


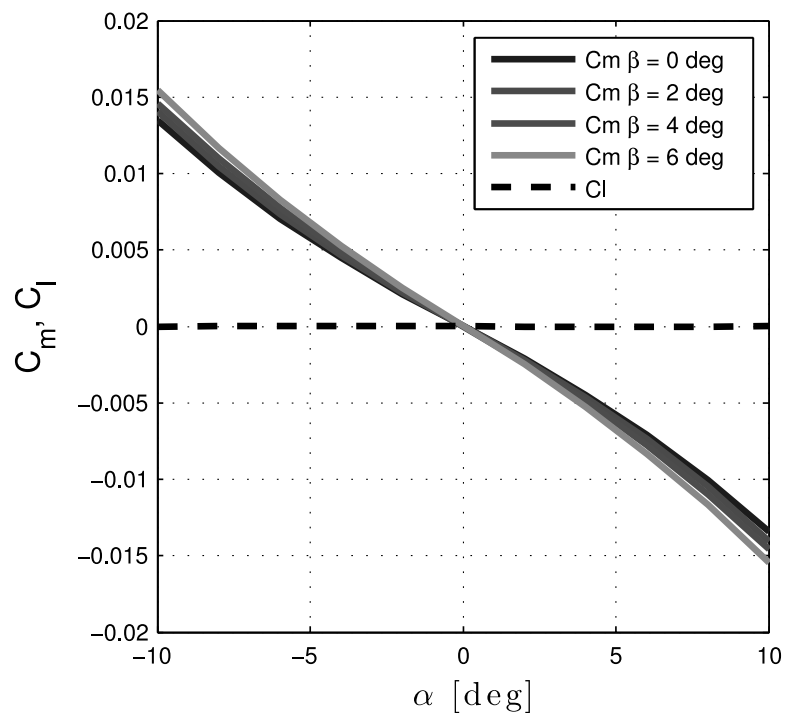

Fig. 2 Variation of $C_{m}$ and $C_{l}$ with angle of attack $(\alpha)$ and sideslip ( $\left.\beta\right)$. Coefficients calculated with $A D B S a t$ using Sentman's model, $S_{r e f}=0.12857 \mathrm{~m}^{2}, L_{r e f}=0.28 \mathrm{~m}$.

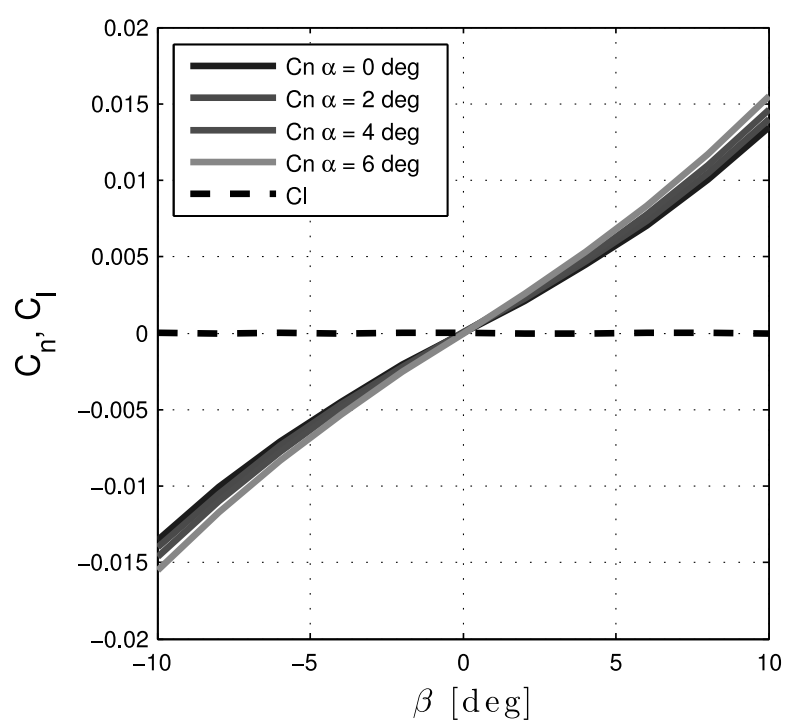

Fig. 3 Variation of $C_{l}$ and $C_{n}$ with sideslip $(\beta)$ and angle of attack $(\alpha)$. Coefficients calculated with ADBSat using Sentman's model, $S_{r e f}=0.12857 \mathrm{~m}^{2}, L_{r e f}=0.28 \mathrm{~m}$.

of the mesh shown in Fig. 1. The variation of $C_{m}$ with $\alpha$ is, for small angles, nearly linear and clearly dominates against the variation of $C_{m}$ with $\beta$, in addition, there is almost no variation of $C_{l}$ with $\alpha$, which is very small compared with $C_{m}$ (Fig. 2). The same is true for the variation of $C_{n}$ and $\beta$ (Fig. 3). All coefficients presented in this paper have been calculated with $A D B S a t$, a 


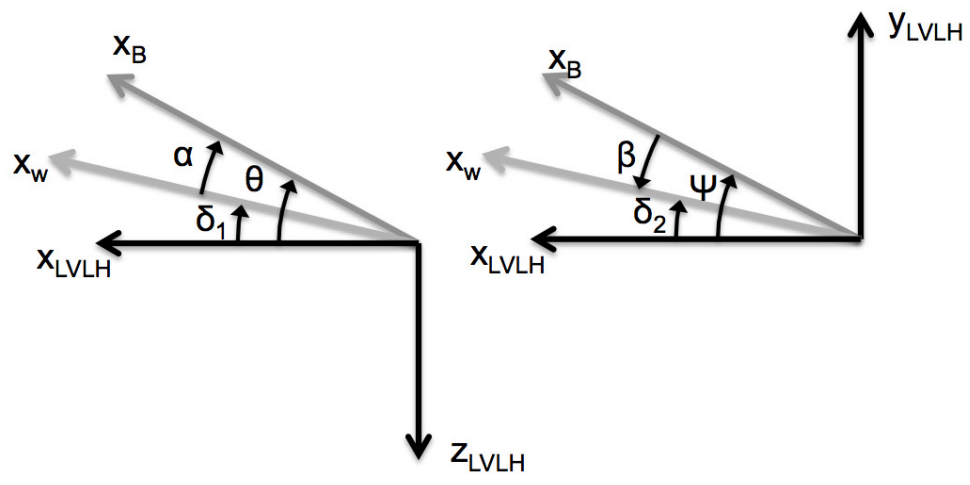

Fig. 4 For small angles, relationship between angle of attack $(\alpha)$, pitch $(\theta)$ and $\delta_{1}$ (left) and sideslip $(\beta)$, yaw $(\psi)$ and $\delta_{2}$ (right)

program that allows for fast calculation of moment and force coefficients for 3D convex geometries in free molecular flow, developed at Manchester University. It incorporates a shadow analysis and the possibility of choosing different force models. By default it should be assumed that all coefficients have been calculated using a diffuse Sentman model, with accommodation coefficient $=1$, speed ratio, $s=4$, and surface and atmospheric temperatures equal to $T_{w}=300 \mathrm{~K}$ and $T_{\text {inf }}=1000 \mathrm{~K}$. We can now relate the Euler and aerodynamic angles. From Fig. 4 we have:

$$
\begin{aligned}
& \alpha=\theta-\delta_{1} \\
& \beta=\delta_{2}-\psi
\end{aligned}
$$

Where $\delta_{1}$ and $\delta_{2}$ are the angles which define the orientation of the aerodynamic velocity in the orbital frame. For a circular orbit, these are the angles between inertial and aerodynamic velocities, as a result of wind and atmospheric rotation, so they are small. Then, the aerodynamic moments are given by:

$$
\begin{gathered}
T_{a x}=0 \\
T_{a y}=\frac{1}{2} \rho V_{a}^{2} S_{r e f} L_{r e f} C_{m_{\alpha}} \theta-\frac{1}{2} \rho V_{a}^{2} S_{r e f} L_{r e f} C_{m_{\alpha}} \delta_{1} \\
T_{a z}=\frac{1}{2} \rho V_{a}^{2} S_{r e f} L_{r e f} C_{n_{\beta}} \delta_{2}-\frac{1}{2} \rho V_{a}^{2} S_{r e f} L_{r e f} C_{n_{\beta}} \psi
\end{gathered}
$$


Using the above expressions we can re-write the rotation equations as:

$$
\begin{gathered}
I_{x} \ddot{\phi}+4 \omega_{0}^{2}\left(I_{y}-I_{z}\right) \phi-\omega_{0}\left(I_{x}-I_{y}+I_{z}\right) \dot{\psi}=T_{d x} \\
I_{z} \ddot{\psi}+\omega_{0}^{2}\left(I_{y}-I_{z}\right) \psi+Q S_{r e f} L_{r e f} C_{n_{\beta}} \psi+\omega_{0}\left(I_{x}-I_{y}+I_{z}\right) \dot{\phi}=T_{d z}{ }^{*} \\
I_{y} \ddot{\theta}+3 \omega_{0}^{2}\left(I_{x}-I_{z}\right) \theta-Q S_{r e f} L_{r e f} C_{m_{\alpha}} \theta=T_{d y}{ }^{*}
\end{gathered}
$$

Where $Q=\frac{1}{2} \rho V_{a}^{2}$. Notice that the aerodynamic moments due to $\delta_{1}$ and $\delta_{2}$ have been included in the disturbance torques $T_{d}{ }^{*}$.

\section{Stability analysis}

In the following analysis the term stability means that Euler angles (roll, pitch and yaw) can be kept arbitrarily small for $t>0$ if we make them and their derivatives sufficiently small in $t=0$ [10]. Notice that with this definition of stability, the torque-free motion of a spacecraft traveling in an orbit is not stable.

\section{A. Pitch Stability}

The characteristic equation of the pitch axis is de-coupled from roll and yaw, so we can perform a separate analysis. By means of Laplace transformation:

$$
I_{y} s^{2}+3 \omega_{0}^{2}\left(I_{x}-I_{z}\right)-Q S_{r e f} L_{r e f} C_{m_{\alpha}}=0
$$

Eq. 20 is an harmonic oscillator $(\ddot{x}+c=0)$ which has unstable roots (positive real part) if $c<0$. Then, the necessary stability condition for the pitch axis becomes:

$$
3 \omega_{0}^{2}\left(I_{x}-I_{z}\right)-Q S_{r e f} L_{r e f} C_{m_{\alpha}}>0
$$

We define now:

$$
\sigma_{y}=\frac{I_{x}-I_{z}}{I_{y}} \quad \sigma_{x}=\frac{I_{y}-I_{z}}{I_{x}} \quad \sigma_{z}=\frac{I_{y}-I_{x}}{I_{z}}
$$

Then,

$$
\sigma_{y}-\frac{Q}{3 \omega_{0}^{2}} \frac{S_{r e f} L_{r e f}}{I_{y}} C_{m_{\alpha}}>0
$$


It can be proven for all $\sigma$ defined in Eq. 22 that $-1<\sigma<1$. We can make use of this fact to establish a sufficient condition for stability. The worst case condition would be the maximum negative value of $\sigma$. Then, the pitch axis is stable if the following condition is met (sufficient but not necessary):

$$
-\frac{Q}{3 \omega_{0}^{2}} \frac{S_{r e f} L_{r e f}}{I_{y}} C_{m_{\alpha}}>1
$$

From Eq. 24 we can derive the following conclusions:

1) We need $C_{m_{\alpha}}<0$ (aero-stability) since the rest of the parameters are positive by definition.

2) Passive aerostabilization is more effective for small bodies. It can be proved that the term $S_{r e f} L_{r e f} / I_{y}$ decreases with increasing size, so it is generally easier to aerostabilize small spacecraft. This is a reasonable assumption due to the characteristics of FMF: for a given geometry and flight conditions, $C_{m_{\alpha}}$ is constant regardless of the size (as long as the proportions are kept).

If condition 23 is satisfied, an oscillatory motion will occur. Using the inverse Laplace transformation the time response would be:

$$
\theta(t)=\frac{T_{d y}}{I_{y} \Omega^{2}}+\left(\theta_{0}-\frac{T_{d y}}{I_{y} \Omega^{2}}\right) \cos \Omega t+\frac{\dot{\theta_{0}}}{\Omega} \sin \Omega t
$$

Where:

$$
\Omega=\sqrt{3 \sigma_{y} \omega_{0}^{2}-Q S_{r e f} L_{r e f} C_{m \alpha} / I_{y}}
$$

The motion is a biased harmonic oscillation with the amplitude depending on the disturbance torque and initial conditions. The way to limit the amplitude is to increase the frequency of oscillation $(\Omega)$. This is naturally accomplished when the satellite decays, as lower altitudes present higher values of dynamic pressure. Regarding design parameters, for an aerostable spacecraft $\left(C_{m_{\alpha}}<0\right)$ the amplitude is controlled by the absolute value of $C_{m_{\alpha}}$. For gravity gradient stabilization, this is controlled by the difference $I_{x}-I_{z}$. 


\section{B. Roll-Yaw stability}

Using Eq. 22, the equation of the coupled yaw-roll by means of Laplace transformation is:

$$
\left[\begin{array}{cc}
s^{2}+4 \omega_{0}^{2} \sigma_{x} & -s \omega_{0}\left(1-\sigma_{x}\right) \\
s \omega_{0}\left(1-\sigma_{z}\right) & s^{2}+\omega_{0}^{2} \sigma_{z}+Q S_{r e f} L_{r e f} C_{n_{\beta}} / I_{z}
\end{array}\right]\left[\begin{array}{l}
\phi(s) \\
\psi(s)
\end{array}\right]=\left[\begin{array}{c}
T_{d x}(s) \\
T_{d z}(s) *
\end{array}\right]
$$

The determinant of the system matrix is of the form:

$$
s^{4}+\omega_{0}^{2} B s^{2}+\omega_{0}^{4} C=0
$$

Where:

$$
\begin{gathered}
B=3 \sigma_{x}+\sigma_{x} \sigma_{z}+1+Q S_{r e f} L_{r e f} C_{n_{\beta}} /\left(\omega_{0}^{2} I_{z}\right) \\
C=4 \sigma_{x} Q S_{\text {ref }} L_{r e f} C_{n_{\beta}} /\left(\omega_{0}^{2} I_{z}\right)+4 \sigma_{x} \sigma_{z}
\end{gathered}
$$

For stability we need non-positive real part in the equation roots. If the solutions of $s^{2}$ are both negative, the solutions of $s^{4}$ would be imaginary and the system will be stable. Solving in $s^{2}$ :

$$
\frac{s^{2}}{\omega_{0}^{2}}=\frac{-B \pm \sqrt{B^{2}-4 C}}{2}
$$

Both roots are negative if the following conditions are fulfilled:

$$
\begin{gathered}
B>0 \\
C>0 \\
B^{2}>4 C
\end{gathered}
$$

For easy of notation $U_{1}=Q S_{r e f} L_{r e f} /\left(\omega_{0}^{2} I_{z}\right)$, then the stability conditions:

$$
\begin{gathered}
3 \sigma_{x}+\sigma_{x} \sigma_{z}+1+U_{1} C_{n_{\beta}}>0 \\
4 \sigma_{x} U_{1} C_{n_{\beta}}+4 \sigma_{x} \sigma_{z}>0 \quad(I I) \\
\left(3 \sigma_{x}+\sigma_{x} \sigma_{z}+1+U_{1} C_{n_{\beta}}\right)^{2}>16\left(\sigma_{x} U_{1} C_{n_{\beta}}+\sigma_{x} \sigma_{z}\right) \quad(I I I)
\end{gathered}
$$

These conditions are shown in Fig. 5 for the case $U_{1} C_{n_{\beta}}=0$ (classic Gravity Gradient problem), 


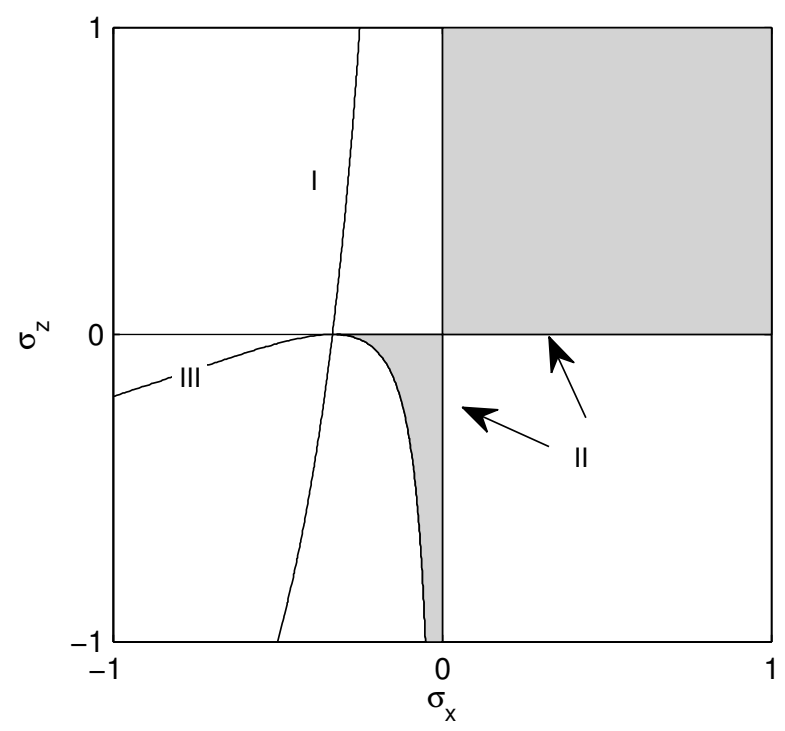

Fig. 5 Conditions I, II and III (Eqs. 35 to 37) for $U_{1} C_{n_{\beta}}=0$. The regions where the values of $\sigma_{x}$ and $\sigma_{z}$ satisfy the three of them are represented in grey.

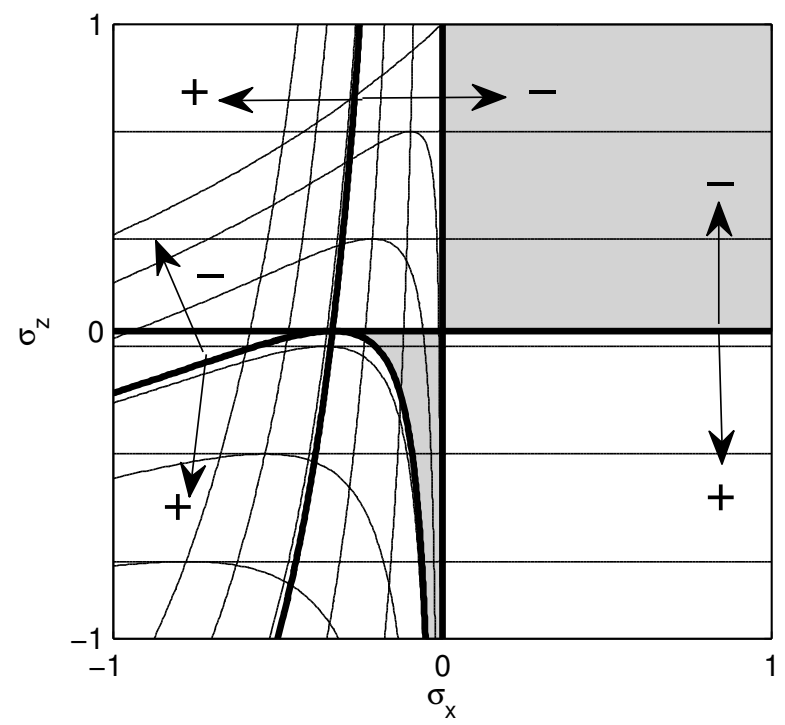

Fig. 6 Variation of the conditions I, II and III (Eqs. 35 to 37) for positive and and negative values of $C_{n_{\beta}}$ (bold lines correspond to $U_{1} C_{n_{\beta}}=0$ ). Notice that the vertical boundary of condition II $\left(\sigma_{x}=0\right)$ is not affected.

grey areas represent the regions where all three conditions are satisfied at the same time. For nonzero values of $U_{1} C_{n_{\beta}}$ the three conditions are modified as is shown in Fig. 6. Positive values of $C_{n_{\beta}}$ push the of condition II horizontal line downwards, increasing the area defined by condition II 
(Eq. 36). Negative $C_{n_{\beta}}$ have the opposite effect, the regions where all three conditions are fulfilled become smaller. To simplify the analysis we transform the above three necessary conditions into two sufficient conditions. From Eq. 36:

$$
4 \sigma_{x}\left(U_{1} C_{n_{\beta}}+\sigma_{z}\right)>0
$$

The worst case condition is $\sigma_{z}=-1$. Therefore, for $U_{1} C_{n_{\beta}}>1$ (which is the same as "pushing" the condition II horizontal line towards $\sigma_{z}=-1$ ) the three conditions are fulfilled in half of the plane (positive $\sigma_{x}$ ). The two sufficient conditions are then:

$$
\begin{gathered}
\sigma_{x}>0 \\
\frac{Q}{\omega_{0}^{2}} \frac{S_{r e f} L_{r e f}}{I_{z}} C_{n_{\beta}}>1
\end{gathered}
$$

Notice that $C_{n_{\beta}}$ needs to be positive in order to satisfy the inequality of Eq. 40 .

In order to visualize all necessary conditions together (1 pitch +3 roll-yaw) we can transfer the pitch condition (Eq. 23) into the $\sigma_{x}-\sigma_{z}$ plane using the following relation:

$$
I_{z}\left(\sigma_{z}-1\right)=I_{x}\left(\sigma_{x}-1\right)
$$

This condition is shown in Fig. 7. For $C_{m_{\alpha}}=0$ (no aerodynamic forces) the condition becomes $\sigma_{z}<\sigma_{x}$ and the stability boundary is $\sigma_{z}=\sigma_{x}$. For negative (stable) $C_{m_{\alpha}}$ the region of stability increases while for positive $C_{m_{\alpha}}$ the region of stability decreases. Adding all four conditions together we can see the regions of stability. Fig. 8 offers the variations of these stability regions with both $C_{m_{\alpha}}$ and $C_{n_{\beta}}$. The first figure (a) shows the pure gravity gradient case without aerodynamic forces, in the second figure (b) both coefficients have a stabilization effect $\left(C_{m_{\alpha}}<0 C_{n_{\beta}}>0\right)$ so the stable region increases, the opposite case is shown in the third one (c), the stability region decreases. The last figure (d) shows the stability region when the three sufficient conditions (Eqs. 24, 39 and 40) are met. Table 1 summarizes these three sufficient conditions for stability. 


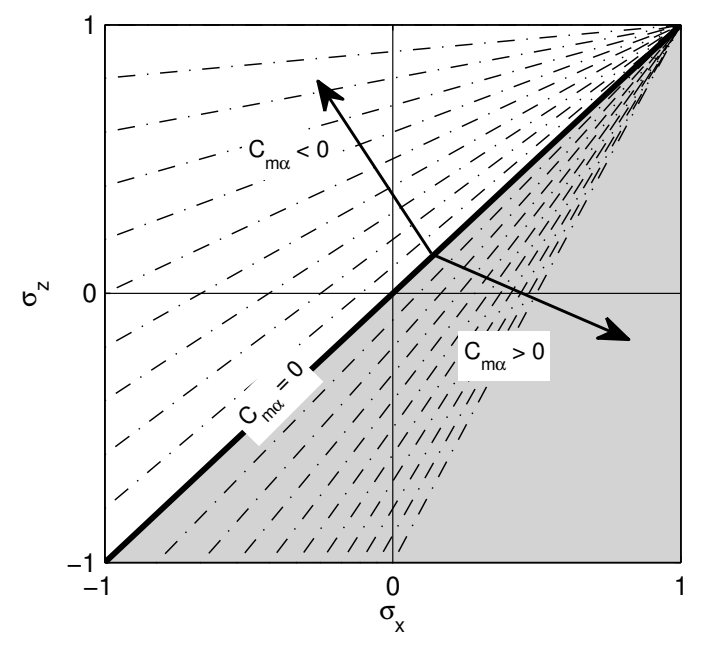

Fig. 7 Variation of the the stability boundary of the pitch condition in the $\sigma_{x}-\sigma_{z}$ plane for positive and negative values of $C_{m_{\alpha}}$. The grey area represents those values of $\sigma_{z}$ and $\sigma_{x}$ compliant with the inequality for $C_{m_{\alpha}}=0$.

(a)

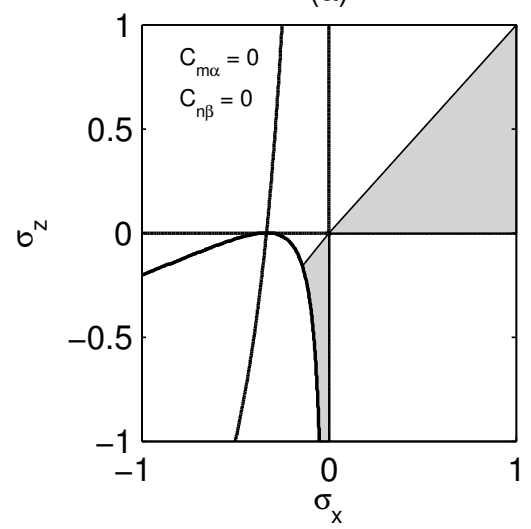

(c)

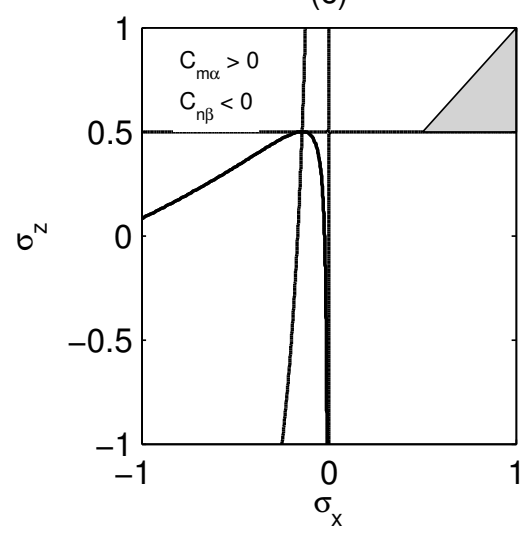

(b)

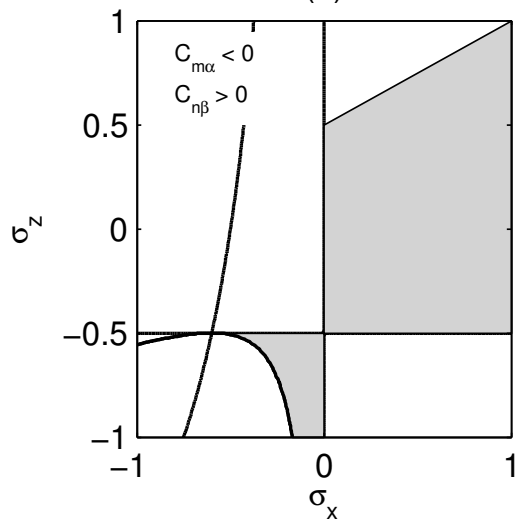

(d)

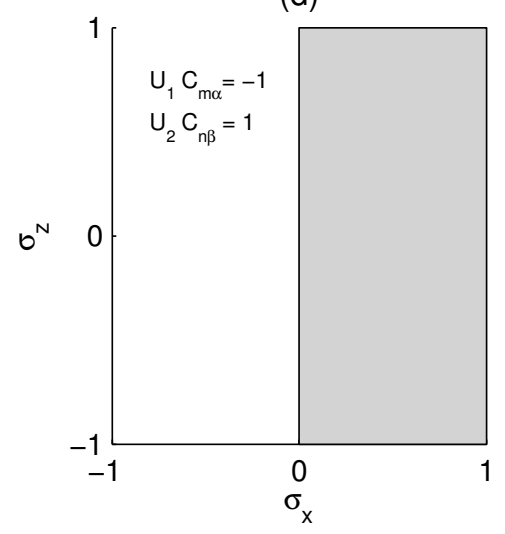

Fig. 8 Roll-yaw stability regions in the $\sigma_{z}-\sigma_{x}$ plane for different values of the stability derivatives $C_{m_{\alpha}}$ and $C_{n_{\beta}}$. 


\begin{tabular}{|l|}
\hline Pitch \\
\hline$-\frac{Q}{3 \omega_{0}^{2}} \frac{S_{r e f} L_{r e f}}{I_{y}} C_{m_{\alpha}}>1$ \\
\hline Roll-Yaw \\
\hline$\frac{Q}{\omega_{0}^{2}} \frac{S_{r e f} L_{r e f}}{I_{z}} C_{n_{\beta}}>1$ \\
$I_{y}>I_{z}$ \\
\\
\end{tabular}

\section{Stability derivatives in Free Molecular Flow}

Stability derivatives can be influenced by design. They are driven by body shape, center of mass location and flight conditions. At orbital altitudes, the calculation of aerodynamic coefficients requires a different approach from aircraft aerodynamics. The flow is characterized by the low density and the small number of collisions between molecules (Free Molecular Flow). Therefore, it can not be considered continuous and classic fluid mechanics theory is no longer applicable. Instead, each molecule interacts individually with the surface wall, exchanging momentum with the body. The kinetic theory of gases predicts these interactions providing analytical expressions to calculate forces for a flat plate under different conditions and angles of incidence (Sentman [31], Schamberg [32], Cook [33]). Those can be applied to each of the elements of a surface mesh representing the geometry of the body. Another approach is the use of numerical codes which directly simulate molecules and its collisions with the surface, like Direct Simulation Monte Carlo, which provide higher fidelity at the expense of calculation time. In the case of complicated geometries, with concave surfaces and the possibility of multiple reflections of the particles, the use of these codes and the extra amount of calculation is justified. However, for trade-off studies, analytical methods able to take into account surface shadowing produce accurate results in a few seconds.

This section studies how the stability derivatives vary with different geometries and flight conditions. To simplify the analysis, the focus is in the pitch momentum or longitudinal axis. However, 
the same analysis is also applicable to the yaw axis. By default it should be assumed that all the coefficients have been calculated using a diffuse Sentman model, with accommodation coefficient $=1$, speed ratio, $s=4$, and surface and atmospheric temperatures equal to $T_{w}=300 \mathrm{~K}$ and $T_{\text {inf }}=1000 K$.

Global vehicle moment coefficients $\left(C_{l}, C_{m}, C_{n}\right)$ are calculated by adding individual mesh elements moment contributions to a common reference point (moment reference centre). We can translate them to the center of mass $(\mathrm{G})$ :

$$
\vec{C}_{M}^{G}=\vec{C}_{M}-\frac{\vec{x}^{G}}{L_{r e f}} \wedge \vec{C}_{F}
$$

Where:

$$
\begin{gathered}
\vec{C}_{M}=\left\{C_{l}, C_{m}, C_{n}\right\} \\
\vec{C}_{F}=\left\{C_{A}, C_{Y}, C_{N}\right\}
\end{gathered}
$$

The coefficients $C_{A}, C_{Y}, C_{N}$ are the axial, lateral and normal force coefficients in body axes. Focusing in the longitudinal axis, for the vehicle to be aero-stable, the slope of $\mathrm{Cm}$ with respect to angle of attack needs to be negative, $C_{m_{\alpha}}<0$, (Fig. 9). That way a restoring torque is created when the angle of attack increases (the contribution of gravity gradient has been treated in previous sections and is neglected here).

Figs. 9 to 12 show the variation of FMF $\mathrm{Cm}$ for different geometries and center of mass positions. They all refer to the base area and diameter $(\mathrm{D})$. The position of the center of mass $\left(x_{\mathrm{cog}}\right)$ is measured from the leading face downstream. Stability is increased by moving the center of mass towards the leading face (steeper $C_{m}$ slopes, lower $C_{m_{\alpha}}$ ). Following the small angle approximation, $C_{m_{\alpha}}$ can be defined as the slope of $C_{m}$ at zero angle of attack (assuming that this angle corresponds to the trim point point of the vehicle). The variation of $C_{m_{\alpha}}$ with center of mass position is linear (Figs. 13 to 14). The change in stability corresponds to the change of sign of $C_{m_{\alpha}}$. Rectangular prisms or cylinders have the point of neutral stability, $C_{m_{\alpha}}=0$, at the geometric center. For more complex geometries, like cones, it depends on the shape and flight conditions. Figs 13 to 14 also show how the stability changes with accommodation coefficient and surface temperature. In both 


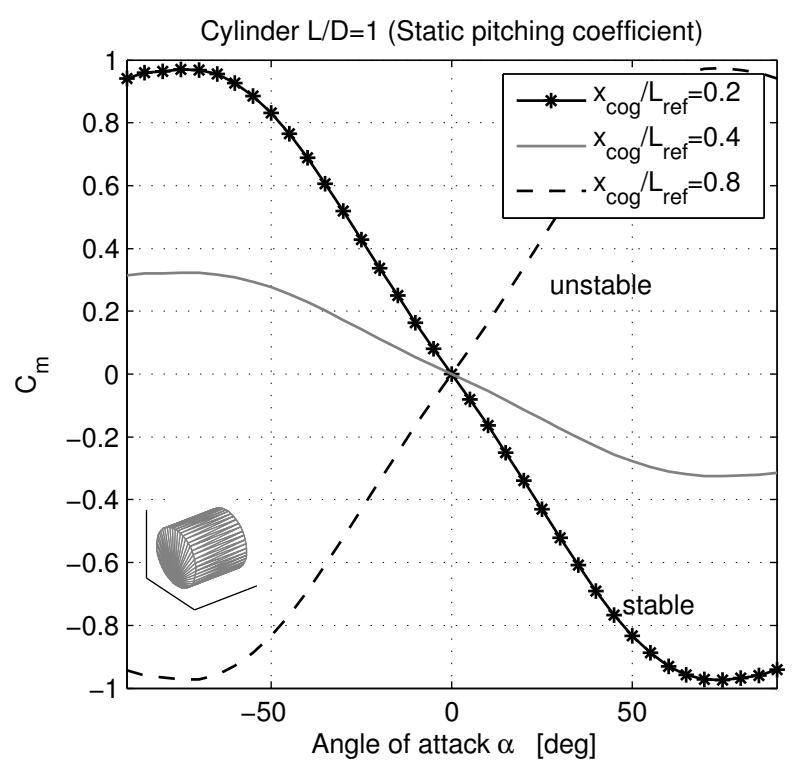

Fig. 9 FMF Static pitching coefficient for a cylinder $(L / D=1)$ referred to the base area and diameter, for various positions of the center of mass.

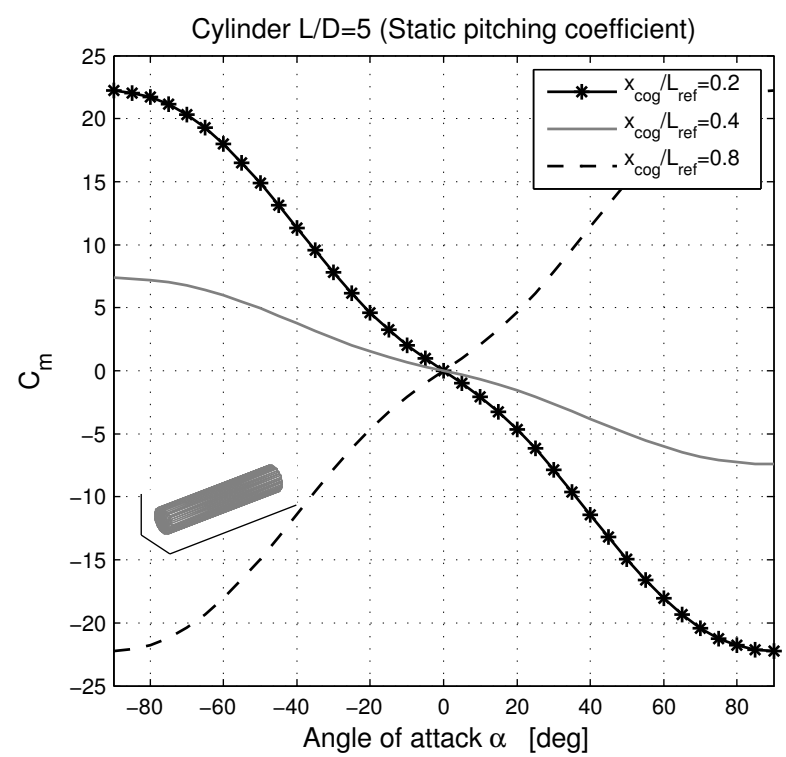

Fig. 10 FMF Static pitching coefficient for a cylinder $(\mathrm{L} / \mathrm{D}=5)$ referred to the base area and diameter, for various positions of the center of mass.

figures it is interesting to note the presence of one center of mass position which is insensitive to accommodation coefficient or surface temperature. The same value of $C_{m_{\alpha}}$ is obtained for different values of these variables. For a rectangular prism or a cylinder (Fig. 13) this point is situated at the geometric center and corresponds to neutral stability $\left(C_{m_{\alpha}}=0\right)$. In the case of the 45 deg cylinder 


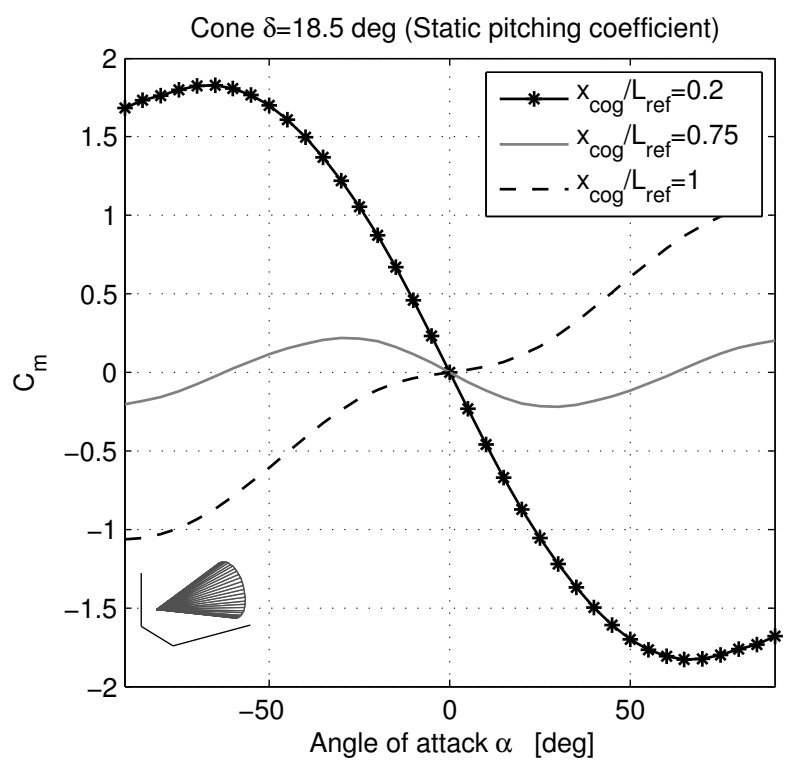

Fig. 11 FMF Static pitching coefficient for a cone (half angle $=18.5 \mathrm{deg}$ ) referred to the base area and diameter, for various positions of the center of mass.

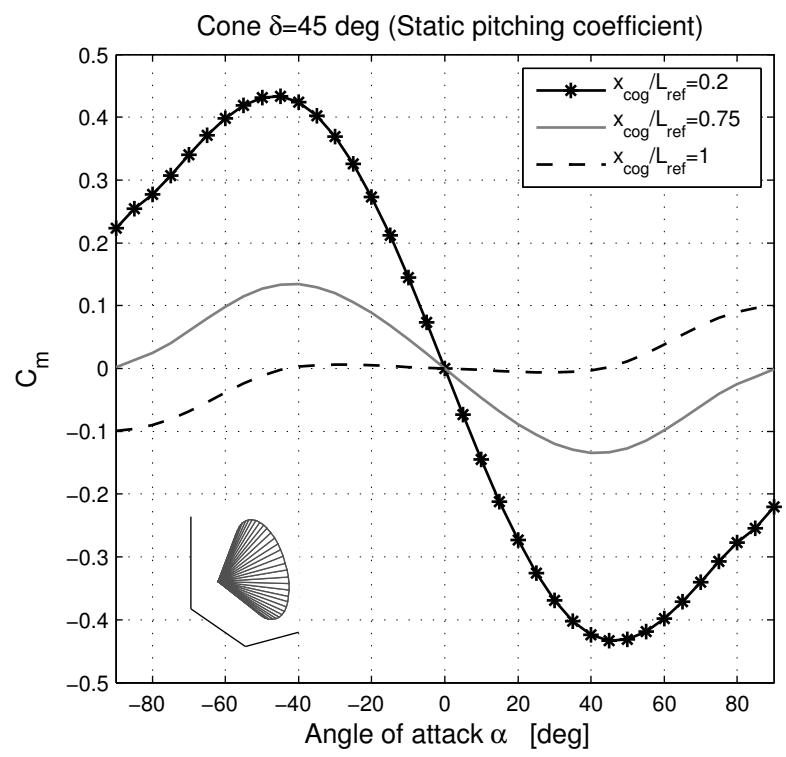

Fig. 12 FMF Static pitching coefficient for a cone (half angle $=45 \mathrm{deg}$ ) referred to the base area and diameter, for various positions of the center of mass.

it is situated around 0.75 and with a value of $C_{m_{\alpha}}$ around -0.6 , both the value and the position of this point are a function of the half angle for cylinders.

Stability in FMF can be completely different from continuous regimes. This effect is well known for re-entry capsules which can become unstable during the free molecular flow phase of the re-entry 

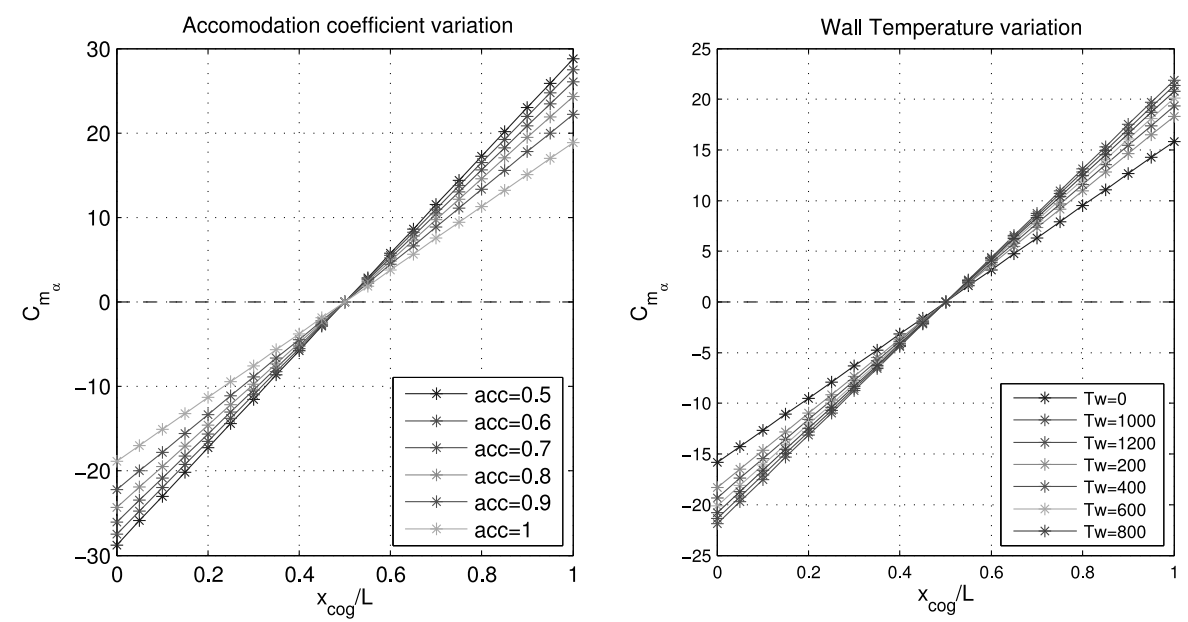

Fig. 13 Cylinder $(\mathrm{L} / \mathrm{D}=5)$. Variation of longitudinal stability derivative $\left(C_{m_{\alpha}}\right)$ with accommodation coefficient (right) and surface temperature (left).
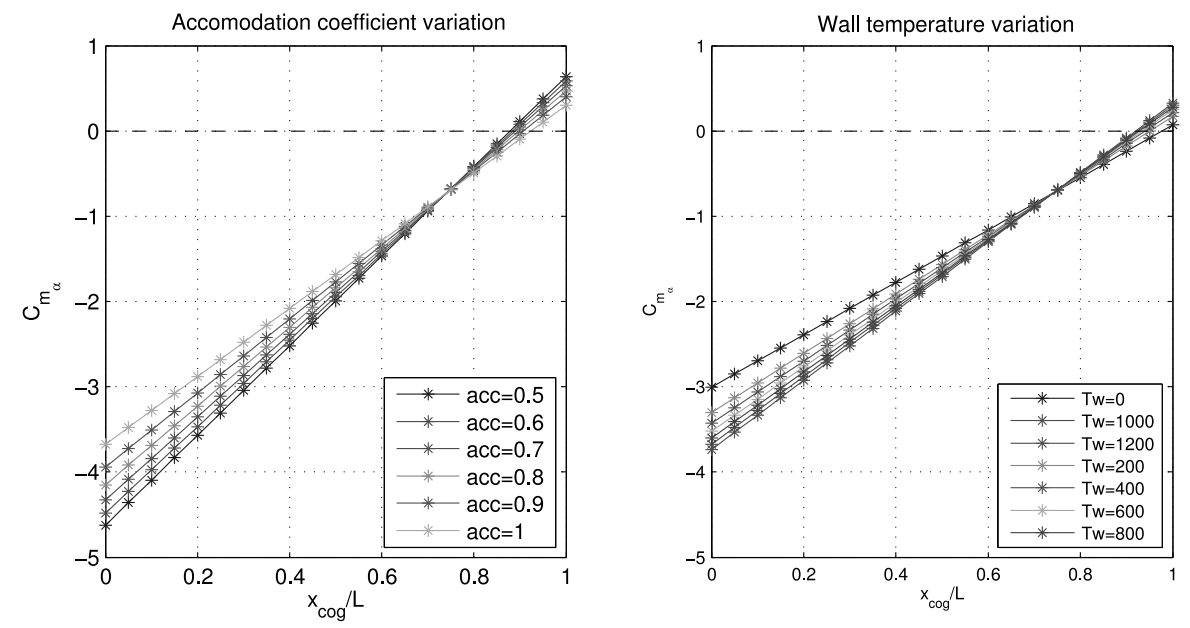

Fig. 14 Cone (half angle $=45 \mathrm{deg}$ ). Variation of longitudinal stability derivative $\left(C_{m_{\alpha}}\right)$ with accommodation coefficient (right) and surface temperature (left).

$([34],[36])$. In Fig. 15, for the same center of mass position the vehicle is stable at hypersonic speed (coefficient calculated using a Newton's method), however unstable in FMF.

Another way to increase the stability is the addition of tail surfaces downstream the center of mass. A shuttlecock configuration of the fins may be appropriate for missions in which it is interesting to increase the drag coefficient (Fig. 16, left), like re-entry or de-orbit. However, for missions in which the satellite lifetime is to be maximized, with this configuration the increase in stability is at the expense of a big increase in drag. Therefore, a "feather" design (Fig. 16, right) 


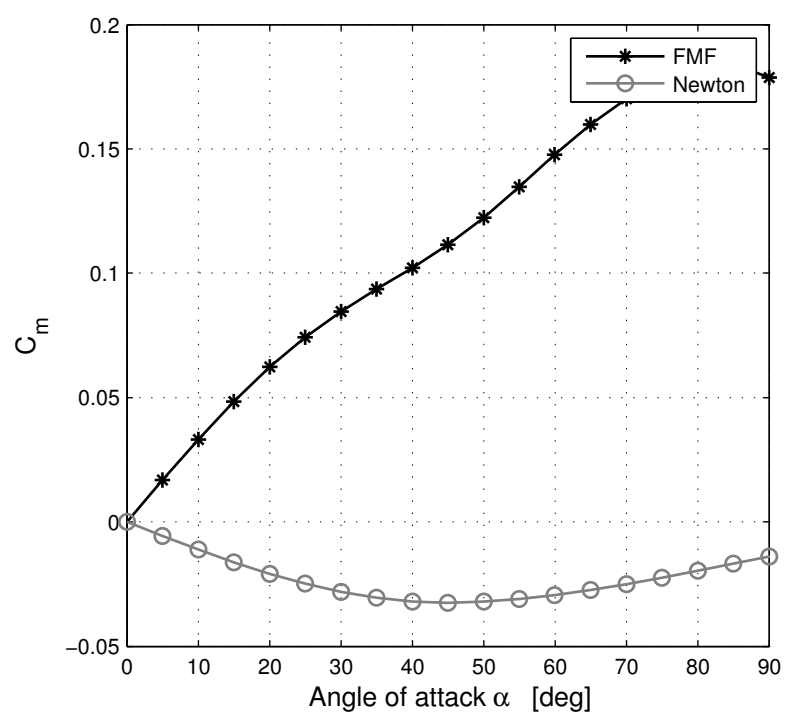

Fig. 15 Cone (half angle $=45 \mathrm{deg}$ ). Different longitudinal stability in FMF (Sentman model) and hypersonic (Newton model) for the same center of mass position.
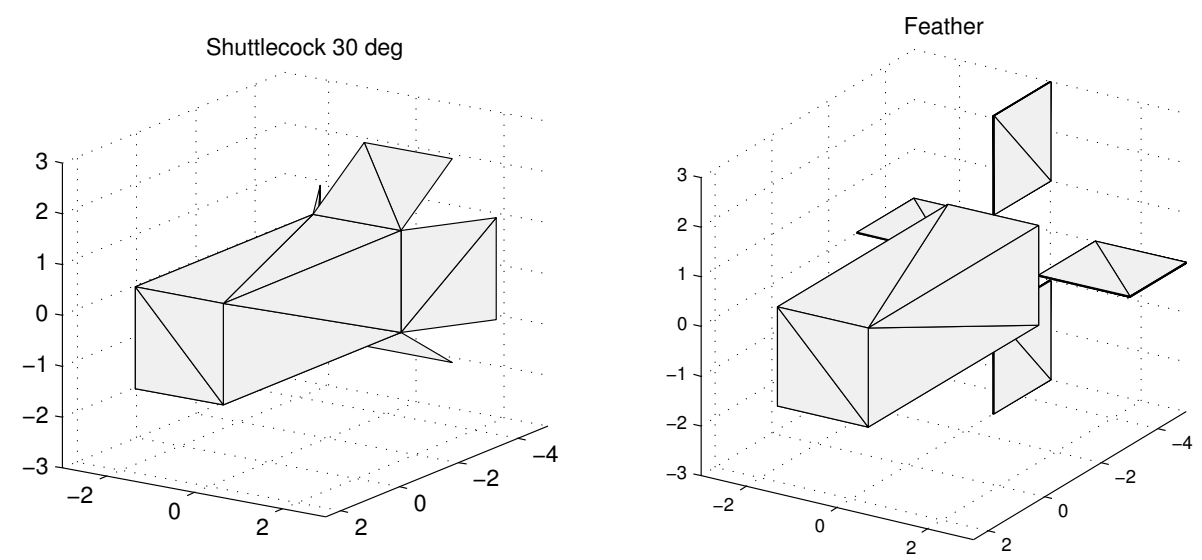

Fig. 16 Shuttlecock (left) and Feather (right) designs

is more convenient. Although it produces a more modest stability improvement, the increment in drag is also smaller. Figs 17 and 18 present increment in $\left(C_{m_{\alpha}}\right)$ and $C_{D}$ for four fins of 1 unit ${ }^{2}$ with respect to the body of a typical $3 \mathrm{U}$ cubesat (rectangular prism of $3 \mathrm{x} 1 \mathrm{x} 1$ ). 


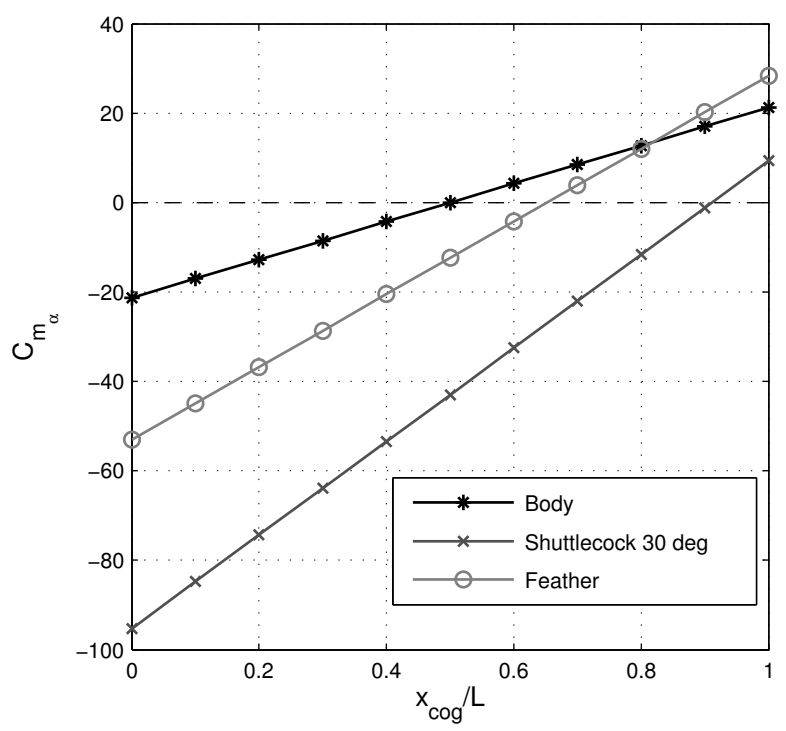

Fig. 17 Stability for rectangular prism (Body) and $1 u^{2}$ fins in Shuttlecock and Feather configurations. All referred to the base area and prism longitude.

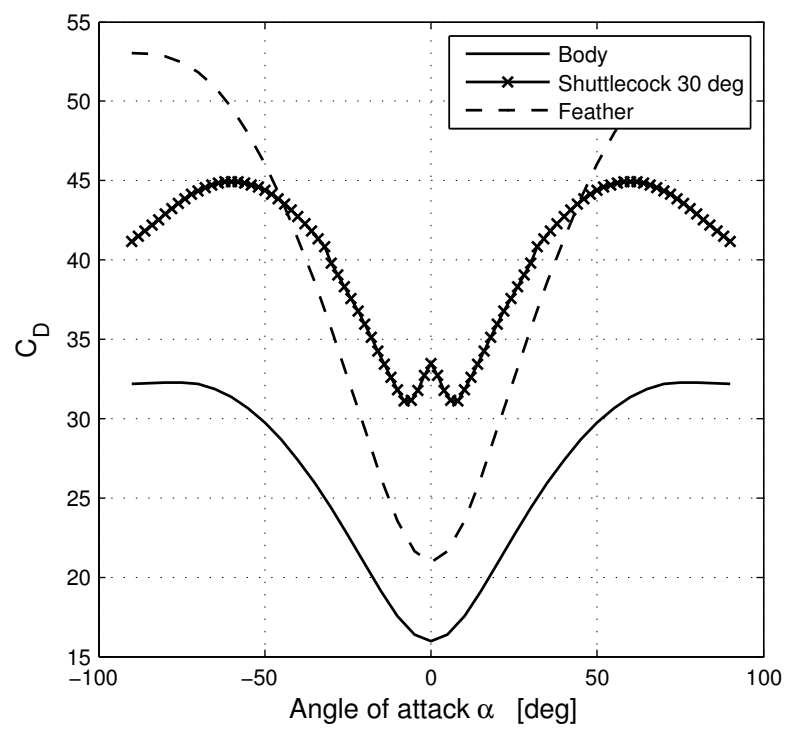

Fig. 18 Drag coefficient for rectangular prism (Body) and $1 u^{2}$ fins in Shuttlecock and Feather configurations. All referred to the base area

\section{Time domain response}

In this section, a complete six degrees of freedom simulation is used to prove the validity of the previous derivation. The simulator includes high fidelity models of various perturbation affecting the problem, such as gravity gradient, aerodynamic forces and torques and atmospheric 


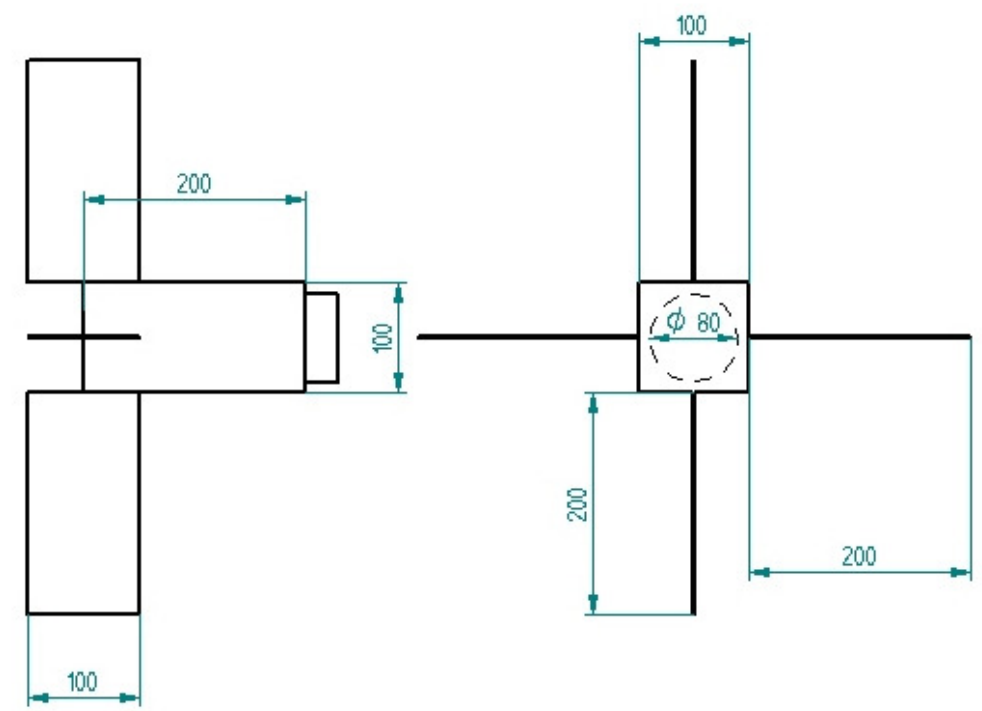

Fig. 19 Geometry of the simulated satellite, units in $\mathrm{mm}$

Table 2 Center of gravity position and estimated $C_{m_{\alpha}}$

\begin{tabular}{lcc}
\hline \hline Case & $x_{\operatorname{cog}}$ & $C_{m_{\alpha}}$ \\
\hline 1 & $0 \%$ & -5.3 \\
2 & $40 \%$ & -2.3 \\
3 & $60 \%$ & -0.77 \\
\hline \hline
\end{tabular}

and wind models (NRLMSISE-00 and HWM-93). FMF coefficients are obtained with ADBSat using Sentman's model with an accommodation coefficient of 1 . This is probably a likely value, exposed surfaces in low earth orbit adsorb atomic oxygen, and due to this contamination accommodation coefficient is nearly independent of the surface material [37]. In all cases, the simulation starts in a circular orbit of $300 \mathrm{~km}$ altitude and $80 \mathrm{deg}$ inclination. Initial attitude angles and inertial rates are zero. This is equivalent to a negative rate in the pitch axis orbital frame (In the absence of an stabilizer torque the pitch angle would therefore drift away from 0). The atmospheric model is set to mean solar and geomagnetic activity $\left(F 10.7=F 10.7_{a v}=140\right.$ and $\left.A_{p}=14,[38]\right)$. The geometry of the simulated satellite has been presented in Fig. 19. 

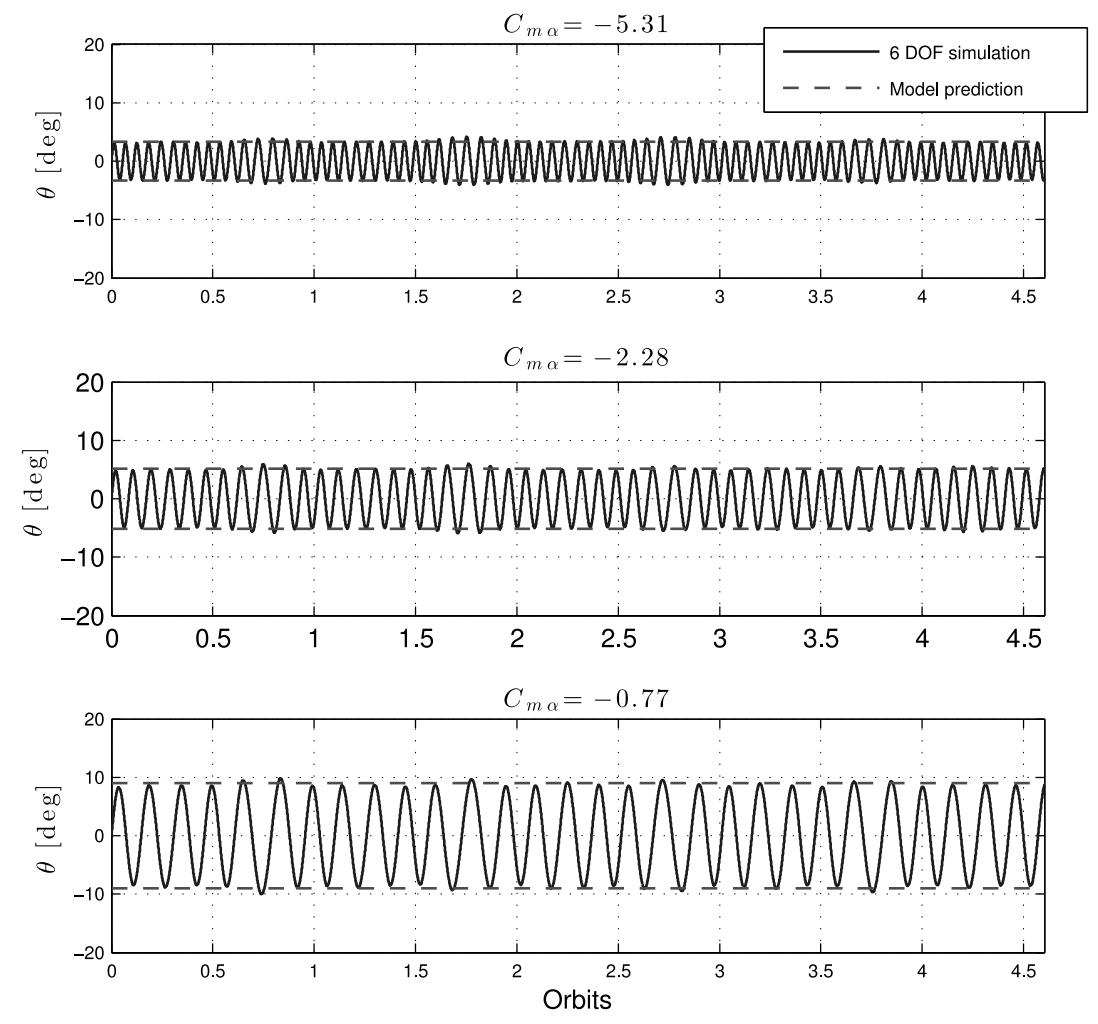

Fig. 20 Non-linear pitch response and amplitude prediction for different values of $C_{m_{\alpha}}$

\section{A. Pitch axis}

To simulate different stability properties, the aerodynamic coefficients are recalculated at different positions of the center of mass along the $\mathrm{x}$ axis (Table 2). Applying Eqs. 24 and 25 it is possible to predict the stability of the motion and the approximate values for the amplitude and frequency. The value of $C_{m_{\alpha}}$ is calculated as the slope of $C_{m}$ at zero angle of attack and zero sideslip and the mean density at $300 \mathrm{~km}$ for mean solar activity is estimated to be $2.52 * 10^{-11} \mathrm{~kg} / \mathrm{m}^{3}$ [38], the inertias for the satellite configuration are $I_{x}=0.005 \mathrm{~kg} \cdot \mathrm{m}^{2}, I_{y}=I_{z}=0.03 \mathrm{~kg} \cdot \mathrm{m}^{2}$. In the absence of an external torque, the amplitude of the oscillation is due to the initial rate in pitch which corresponds to the initial orbital rate. In all three cases Eq. 24 is satisfied, therefore a stable motion is expected. The goal is to understand the sensitivity of the predictions (which are based on simplified assumptions) to real effects such as non-uniform density or non-linear dynamics. Figs 20 and 21 offer the results of the simulations for the pitch evolution. As predicted, the attitude motion is stable and both amplitude and frequency are well correlated with the model predictions. 


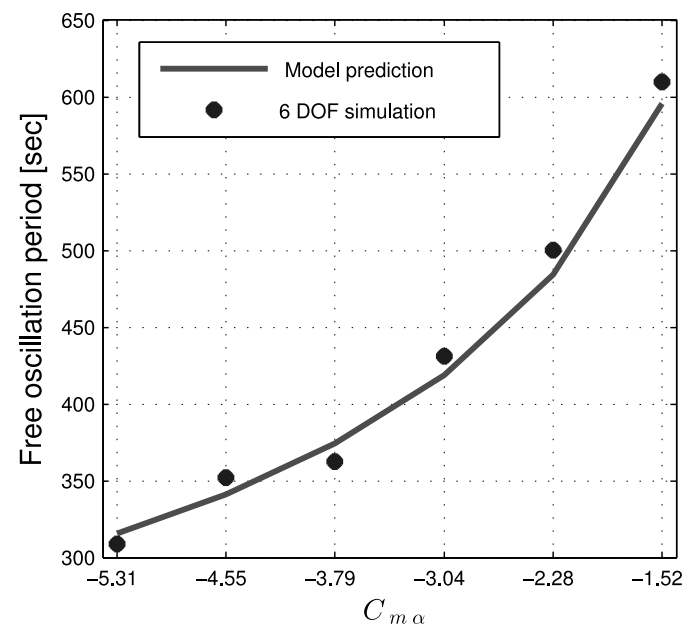

Fig. 21 Predicted and measured period of pitch oscillations for different values of $C_{m_{\alpha}}$

Decreasing the value of $C_{m_{\alpha}}$, increases the amplitude and decreases the frequency of oscillation.

\section{B. Roll-Yaw axis}

Regarding the roll-yaw response, all the sufficient conditions for stability except for the last one $\left(I_{y}>I_{z}\right)$ are satisfied. Therefore, the problem is described by Fig. 8-d. Pitch and yaw axes are stable and the stability of roll motion depends on the difference $I_{y}-I_{z}$. Fig. 22 offers the roll angle for three different values of $\sigma_{x}$, negative (unstable), zero (marginally stable), and positive (stable). Fig. 23 and Fig. 24 show roll, pitch yaw for stable and unstable cases. Notice that despite the instability in roll, both pitch and yaw remain stable. The roll motion interchanges body axes $I_{y}-I_{z}$, however, these are both stable, so the stability of these axes is not compromised in the case of large roll excursions. The co rotating wind can be observed in the low frequency oscillation present in the yaw response, corresponding to the orbital frequency. 


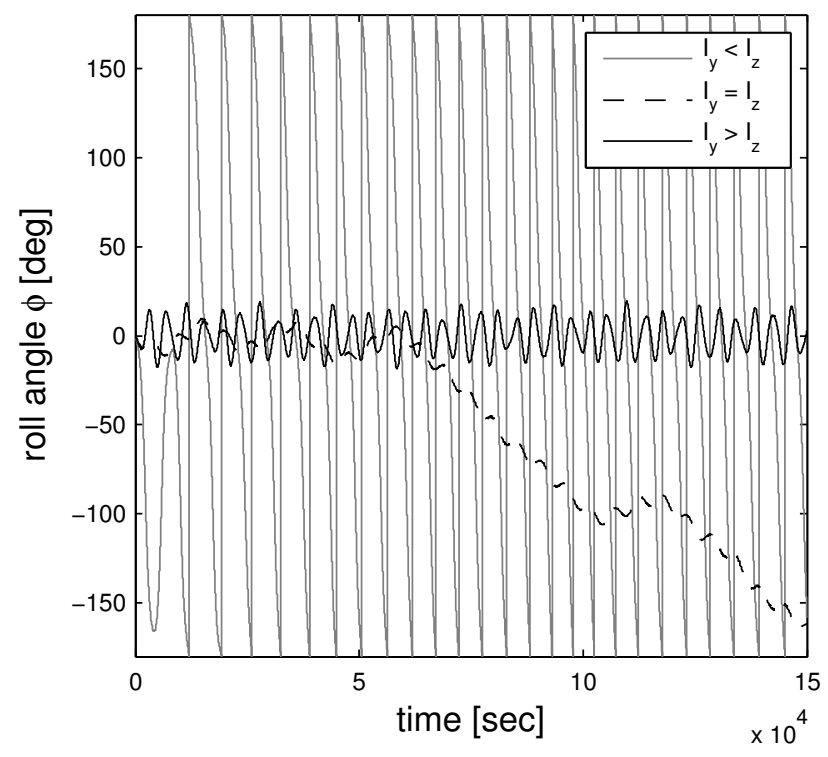

Fig. 22 Roll angle for three different values of $\sigma_{x}$, stable, marginally stable and unstable cases.

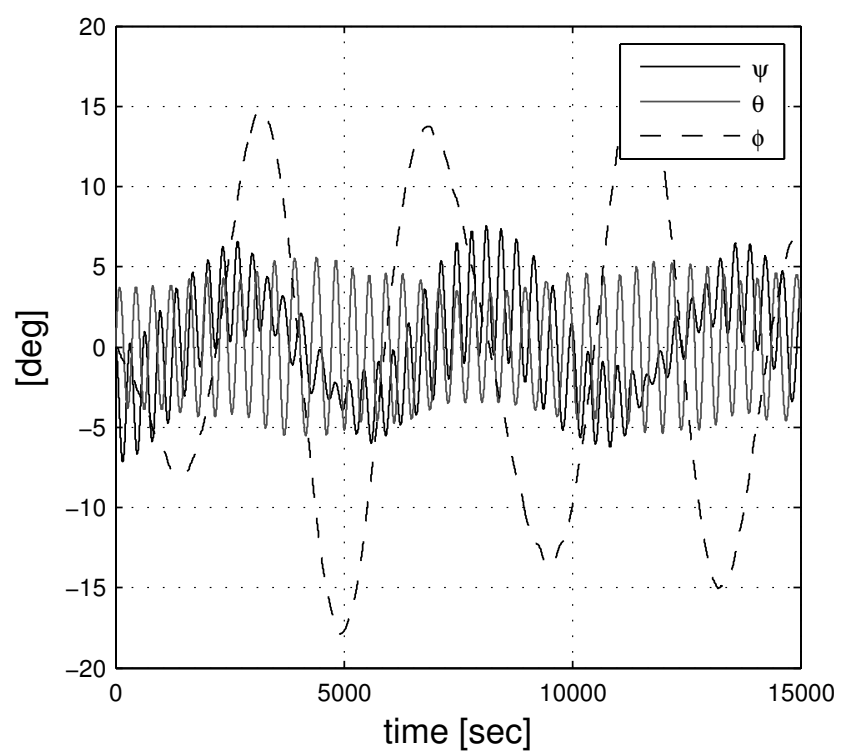

Fig. 23 Roll, pitch, yaw for the stable case. $I_{x}=0.004 I_{y}=0.01, I_{z}=0.008$. 


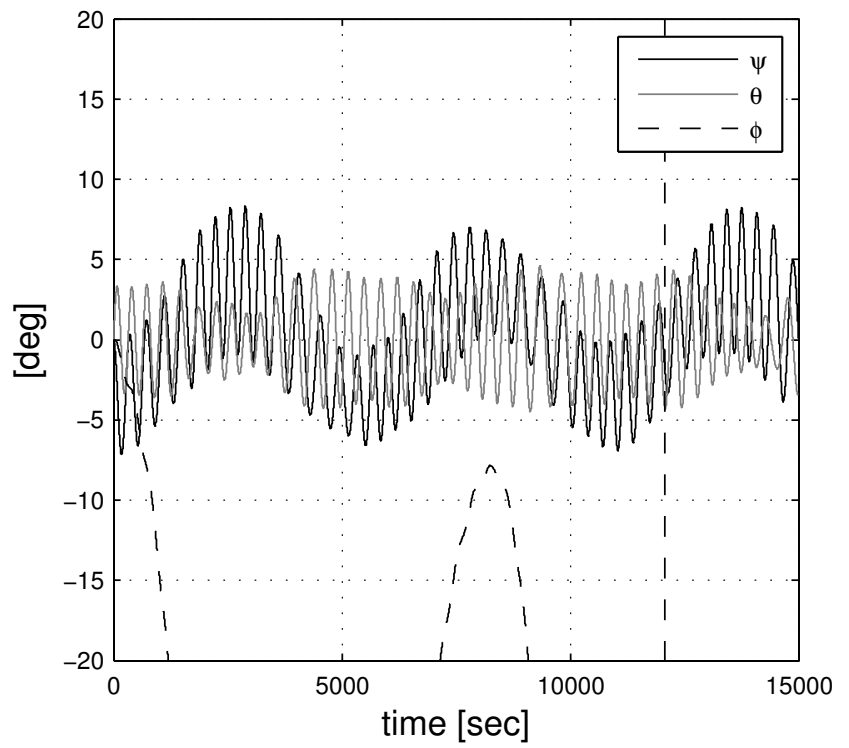

Fig. 24 Roll, pitch, yaw for the roll unstable case. $I_{x}=0.004 I_{y}=0.008, I_{z}=0.01$. 


\section{Stability with varying dynamic pressure}

So far our analysis has considered that all the coefficients in the equations are fairly constant with time. This assumption is valid for quantities such as inertia, $S_{r e f}$ or even $C_{m_{\alpha}}$ for circular orbits. However, it is clear that dynamic pressure has an important time variation due to both density and relative velocity profiles through the orbit. Since the pitch axis is, at least to first order, decoupled from roll-yaw motion we can better understand the effect by looking to the pitch equation:

$$
I_{y} \ddot{\theta}+3 \omega_{0}^{2}\left(I_{x}-I_{z}\right) \theta-q(t) S_{r e f} L_{r e f} C_{m_{\alpha}} \theta=0
$$

Here, $q=q(t)=0.5 \rho(t) V(t)^{2}$, so the dynamic pressure has some sort of variation with time. To characterize this function, we can neglect any effect on the trajectory (drag force, $J_{2} \ldots$ ) as well as any long term variation of the atmosphere (such as variations of solar or geomagnetic activities). For a small number of revolutions these effects are small, as attitude dynamics have a smaller time scale than trajectory dynamics. In such conditions we can assume the dynamic pressure to be a periodic function, $q(t)=q\left(t+T_{\text {orb }}\right)$ where $T_{\text {orb }}$ is the orbital period. Figs. 25 and 26 present variation of dynamic pressure for several altitudes at a given inclination as well as for several inclinations at a given altitude. Both figures have been produced using NRLMSIS-00 with medium solar activity conditions and include atmospheric co-rotation.

Then, it is possible to approximate $q(t)$ analytically by means of Fourier coefficients:

$$
q(t)=q_{0}+\sum_{k=1}^{n} a_{k} \cos \left(k \omega_{0} t\right)+\sum_{k=1}^{n} b_{k} \sin \left(k \omega_{0} t\right)
$$

Where $\omega_{0} T_{\text {orb }}=2 \pi$. As an example, Fig. 27 presents a least-square fitting of the Fourier coefficients for various truncated orders $(n)$.

In such conditions, the equation governing the pitch motion is a special case of second order differential equation with periodic coefficients known as Hill's equation:

$$
\frac{d^{2} y}{d t^{2}}+f(t) y=0
$$

Where $f(t)$ is a periodic function of period $T_{\text {orb }}$. This type of system can exhibit parametric resonance phenomena. For certain values of the parameters, the amplitude of oscillation steadily 


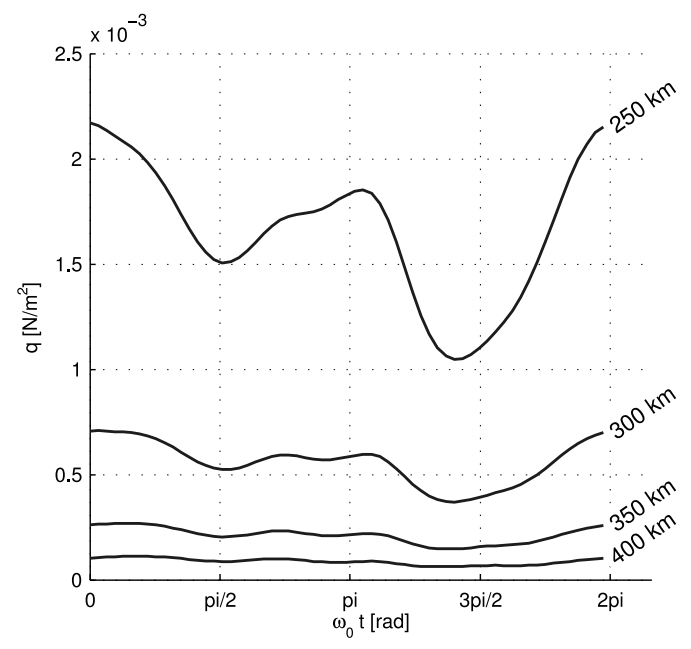

Fig. 25 Variation of dynamic pressure $(q)$ for a circular orbit (80 deg inclination) and different altitudes, starting at the ascending node using NRLMSIS-00 and co-rotating wind

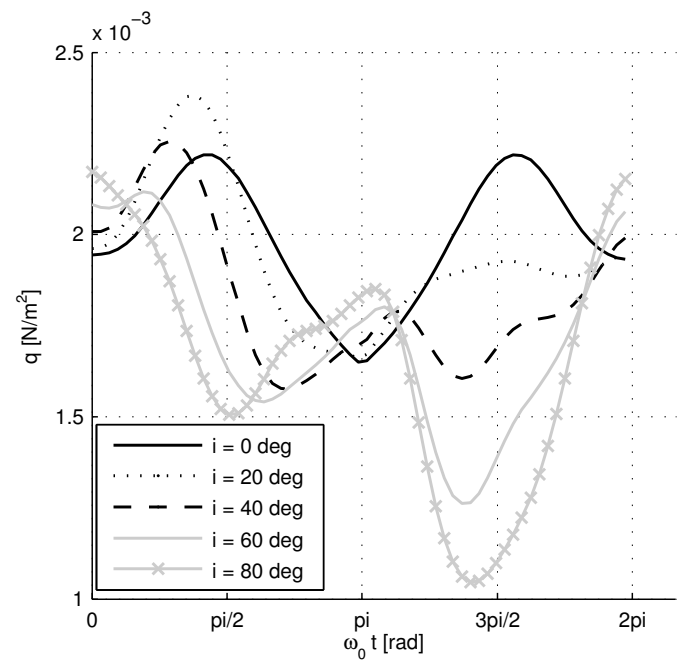

Fig. 26 Variation of dynamic pressure $(q)$ for a circular orbit (250 $\mathrm{km}$ altitude) and different inclinations, starting at the ascending node using NRLMSIS-00 and co-rotating wind

increases leading to instability. A classic example of analysis of such systems is the Mathieu equation, used extensively to model different phenomena in physics and engineering [39]:

$$
\frac{d^{2} y}{d t^{2}}+(\delta-2 \epsilon \cos (2 t)) y=0
$$

Here, the quantities $\delta$ and $\epsilon$ are constants. The analysis consists of finding regions of stability and instability on the $\delta-\epsilon$ plane. These plots are called stability charts. Fig 28 shows a typical stability chart for the Mathieu equation. Kumar et al. [21] used these Mathieu stability charts 


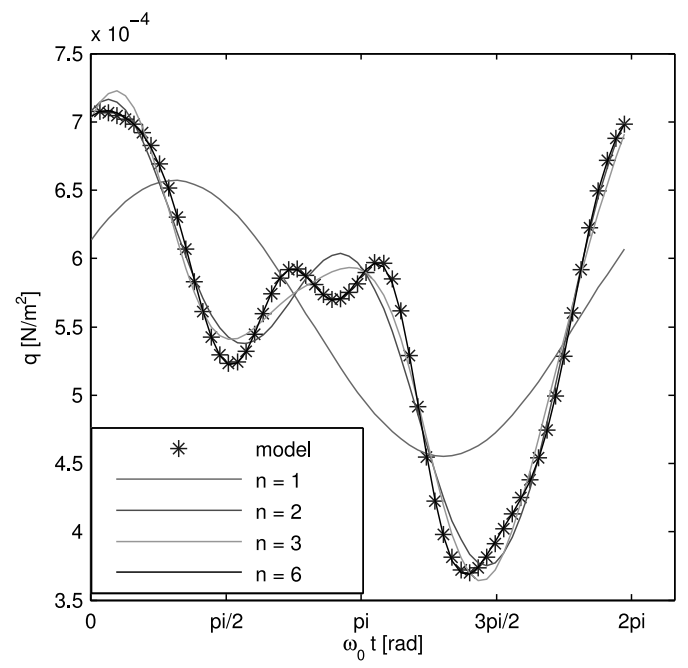

Fig. 27 Fitting of dynamic pressure using a truncated Fourier series of order $n$. Circular orbit (300 km altitude, $80 \mathrm{deg}$ inclination)

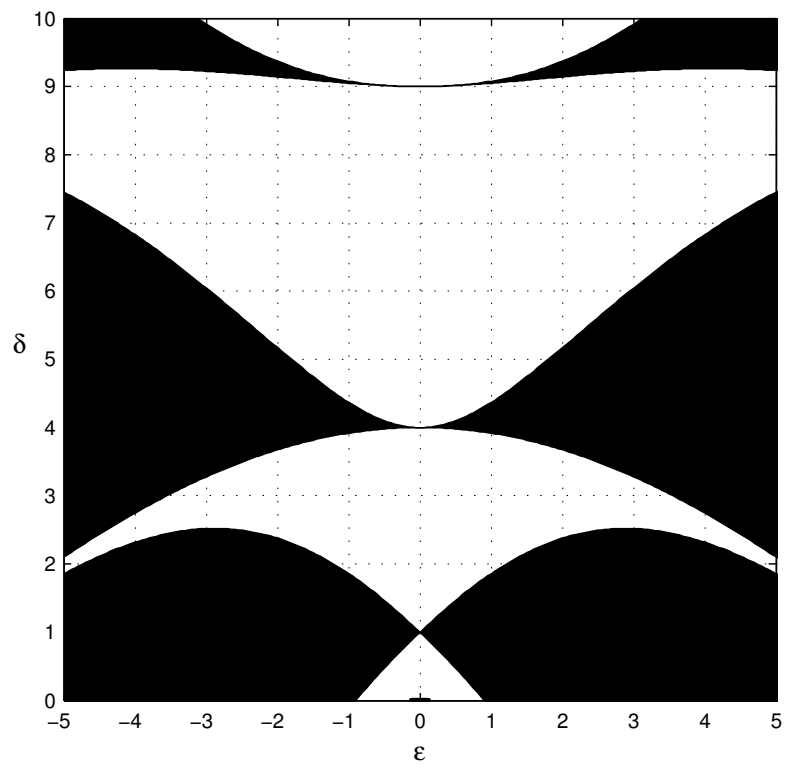

Fig. 28 Mathieu equation stability chart. Black areas are regions of instability

to analyze parametric resonance phenomena on the PAMS satellite. They used a simplified orbit variation of the density (a cosine function) so the system can be simplified to a Mathieu equation. Higher harmonics are treated separately by assuming one frequency at a time, obtaining this way a superimposition of multiple Mathieu diagrams. By design, the PAMS satellite had all three inertia moments equal, so no gravity gradient torque is considered in the analysis. 
To analyze the problem via the Mathieu chart, we need to simplify the dynamic pressure to a cosine function:

$$
q(t)=q_{0}+a_{1} \cos (w t)
$$

Then perform a change of independent variable in the form $\xi=w t / 2$, this way our $\delta$ and $\epsilon$ are:

$$
\begin{gathered}
\delta=12 \sigma_{y}-\frac{4 \Gamma_{g} q_{0}}{\omega_{0}^{2}} \\
\epsilon=\frac{2 \Gamma_{g} a_{1}}{\omega_{0}^{2}}
\end{gathered}
$$

Where

$$
\begin{gathered}
\sigma_{y}=\frac{I_{x}-I_{z}}{I_{y}} \\
\Gamma_{g}=\frac{S_{r e f} L_{r e f} C_{m_{\alpha}}}{I_{y}}
\end{gathered}
$$

This method can be useful to check wether we cross instability regions during the mission. Obtaining, for instance, the evolution of $\left(q_{0}, a_{1}\right)$ values as the altitude decreases, and then plotting the path followed on the Mathieu chart. Crossing a region of instability may not be an issue as long as we remain in the stable region most of the mission. However, as Kumar et al. also observed higher frequency terms may not be negligible, having an impact on stability. Also, the inclusion of other effects, such as wind is not straightforward. To perform a more complete analysis, we use numerical method based on Floquet theory [40]. It allows the stability of the whole system to be analyzed at once, avoiding the splitting of the multiple dynamic pressure frequencies while considering a realistic profile of dynamic pressure, gravity gradient effects and atmospheric winds. The method consists on finding the eigenvalues of the fundamental solution matrix $(H)$ which has the following form:

$$
H=\left(\begin{array}{ll}
\theta_{i}\left(t=T_{\text {orb }}\right) & \dot{\theta}_{i}\left(t=T_{\text {orb }}\right) \\
\theta_{j}\left(t=T_{\text {orb }}\right) & \dot{\theta}_{j}\left(t=T_{\text {orb }}\right)
\end{array}\right)
$$

The values of $\theta\left(t=T_{\text {orb }}\right)$ and $\dot{\theta}\left(t=T_{\text {orb }}\right)$ are obtained by numerically integrating the equation for one period $(0-T)$ for two different initial conditions:

$$
\left(\begin{array}{c}
\theta_{i}(0) \\
\dot{\theta}_{i}(0)
\end{array}\right)=\left(\begin{array}{l}
1 \\
0
\end{array}\right)
$$




$$
\left(\begin{array}{c}
\theta_{j}(0) \\
\dot{\theta}_{j}(0)
\end{array}\right)=\left(\begin{array}{l}
0 \\
1
\end{array}\right)
$$

Then, if any eigenvalue of $H$ has a real part with absolute value greater than $1\left(\left|\lambda_{n}\right|>1\right)$ the system is unstable and the solutions are not bounded. To obtain the regions of potential instability, the method needs to be applied to each of the points of a grid of equation parameters. Other numerical methods can be used to check stability. However, they often require integrating for a longer number of periods and the definition of a ratio between initial and final amplitudes, which is not always clear $([41],[42])$.

In this case, the stability condition has to be evaluated for each pair of $\delta-\epsilon$ values, which is computationally intensive. Before applying the method to the problem, Eq. 45 needs to be re-arranged as follows:

$$
\ddot{\theta}+\left[3 \omega_{0}^{2} \sigma_{y}-\Gamma_{g}\left(q_{0}+\sum_{k=1}^{n} a_{k} \cos \left(k \omega_{0} t\right)+\sum_{k=1}^{n} b_{k} \sin \left(k \omega_{0} t\right)\right)\right] \theta=0
$$

All the parameters in Eq. 57 except for $\sigma_{y}$ and $\Gamma_{g}$ are defined for a given orbit and environmental conditions. Altitude, inclination, epoch and environmental conditions (solar and geomagnetic activity) define the parameters of the fitting of dynamic pressure $\left(q_{0}, a_{k}\right.$ and $\left.b_{k}\right)$. Then, for a given orbit and conditions it is possible to study which values of $\sigma_{y}$ and $\Gamma_{g}$ (geometric parameters) can lead to parametric resonance. The value of $C_{m_{\alpha}}$ is also influenced by the altitude and atmospheric composition, but this is not taken into account for simplicity. Notice that these stability charts are different from the Mathieu ones previously presented and used in other references. In those, the values of the constants $\delta-\epsilon$ contain geometric quantities and flight conditions. Here we fix the flight condition, so the charts depend only on geometric properties of the satellite, quantities that can be influenced by design, such as inertia or $C_{m_{\alpha}}$.

Figures 29 and 30 present some stability charts obtained for different inclination and altitude conditions, using medium solar activity. Black areas are the regions of potential instability. The unstable area at the top of the graphs corresponds to the static stability already predicted by the previous analysis. Black bands are regions of parametric resonance, which correspond to different 

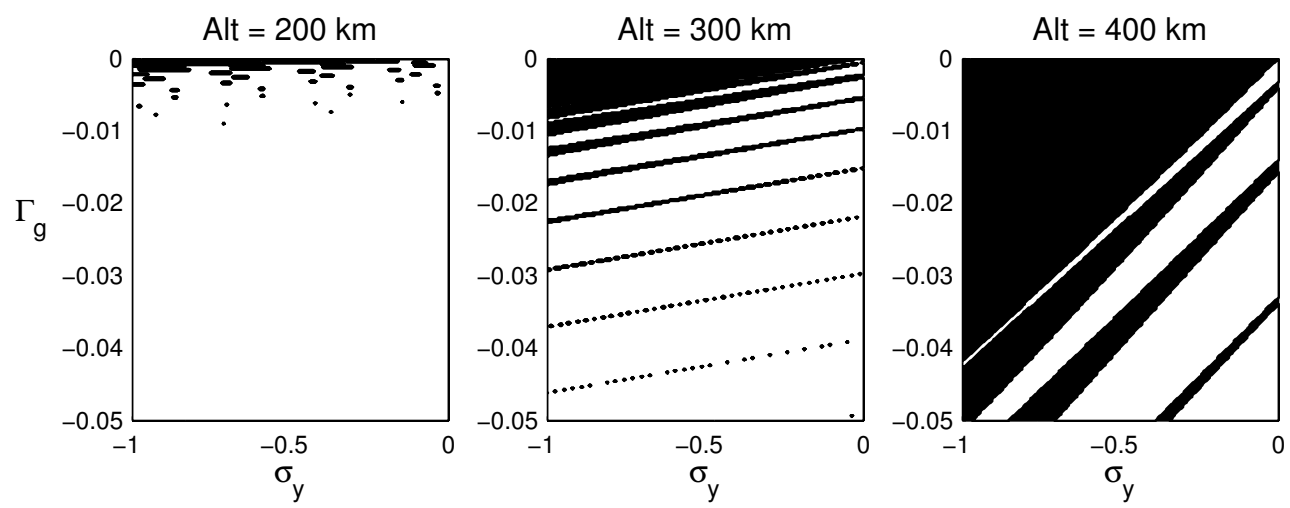

Fig. 29 Evolution of pitch stability chart for a circular orbit of 45 deg inclination and different altitudes
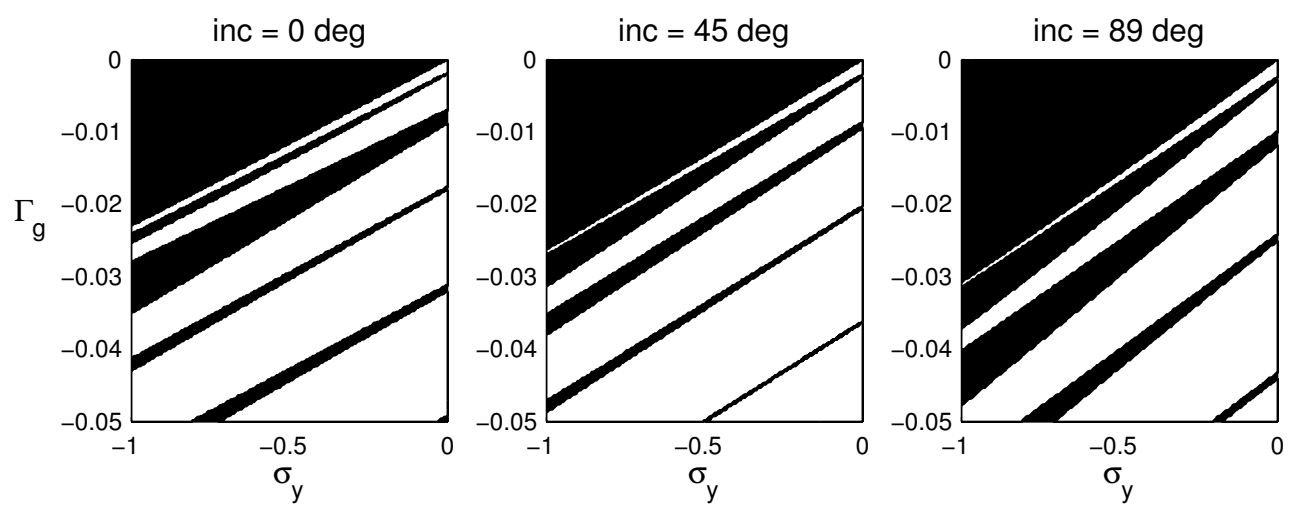

Fig. 30 Evolution of pitch stability chart for a circular orbit of $350 \mathrm{~km}$ and different inclinations

frequencies of the dynamic pressure variation. As the altitude increases, the density decreases and also the regions of instability. The trend in inclination is more difficult to identify, however, higher inclinations present a lower mean dynamic pressure and more variation along the orbit (as a result of altitude variations caused by oblate Earth shape) which is reflected by the growth of both the static instability area and the potential parametric resonance bands. Low or high solar activity would effectively decrease or increase respectively the altitudes at which parametric resonance is likely to occur.

\section{Conclusions}

We have proposed a way to analyze the stability of a satellite subjected to Free Molecular aerodynamic and gravity gradient torques. This is particularly interesting for missions flying in Low 
Earth Orbits. Apart from the benefits of a better understanding, an active design of the satellite stability characteristics can reduce attitude control requirements both by better sizing environmental torques and by minimizing the amount of active control. It can also help characterizing and avoiding potential resonance phenomena.

It has been shown that for phases with low pointing requirements (Cubesats, drag de-orbit devices), attitude stability can even be achieved without any active control, reducing complexity and cost. Other interesting potential applications are the characterization of the atmospheric environment based on measured attitude response or the use of aerodynamic moments to perform slow attitude maneuvers or damp momentum. In either case, this analysis provides a theoretically robust approach for attitude control design.

This study is aplicable to orbits in which Free Molecular Flow can be assumed, (typically form $160 \mathrm{~km}$ altitude and above). For altitudes leading to re-entry in which hypersonic flow is a more reasonable assumption the lack of damping would need to be revised.

\section{References}

[1] "Spacecraft aerodynamic torques," Tech. rep., NASA SP-8058, 1971.

[2] Moe, K. and Reiter, G. S., "Surface-Particle-Interaction Measurements Using Paddlewheel Satellites," Tech. rep., Douglas Aircraft CO inc, Santa Monica CA, Space sciences dept, 1968.

[3] Moe, K., "Absolute atmospheric densities determined from the spin and orbital decays of Explorer VI," Planetary and Space Science, Vol. 14, No. 11, 1966, pp. 1065-1075.

[4] "OSO 1 Orbiting Solar Observatory Satellite," Tech. rep., NASA SP-57, 1965.

[5] "Proceedings of the Symposium on Passive Gravity-Gradient Stabilization," Tech. rep., NASA SP-107, 1966.

[6] "Proceedings of the Symposium on Gravity Gradient Attitude Stabilization," Tech. rep., Aerospace Corporation, El Segundo, California, 1968.

[7] White, J. S., Shigemoto, F. H., and Bourquin, K., "Satellite attitude control utilizing the Earth's magnetic field," Tech. rep., NASA TND-1068, 1961.

[8] Kamm, L., "Magnetorquer-A Satellite Orientation Device," ARS Journal, Vol. 31, No. 6, 1961, pp. $813-815$.

[9] Fischell, R. E., "Magnetic damping of the angular motions of earth satellites," ARS Journal, Vol. 31, 
No. 9, 1961, pp. 1210-1217.

[10] Kane, T. R., Likins, P. W., and Levinson, D. A., Spacecraft dynamics, Vol. 1, New York, McGraw-Hill Book Co, 1983, 445 p., 1983.

[11] Sidi, M. J., Spacecraft dynamics and control: a practical engineering approach, Vol. 7, Cambridge university press, 1997.

[12] De Bra, D., "The Effect of Aerodynamic Forces on Satellite Attitude," Journal of Astronautical Sciences, Vol. 6, 1959, pp. 40-45.

[13] Meirovitch, L. and WALLACE, F., JR, "On the effect of aerodynamic and gravitational torques on the attitude stability of satellites." AIAA Journal, Vol. 4, No. 12, 1966, pp. 2196-2202.

[14] Ravindran, R. and Hughes, P., "Optimal aerodynamic attitude stabilization of near-Earth satellites," Journal of Spacecraft and Rockets, Vol. 9, No. 7, 1972, pp. 499-506.

[15] Schrello, D., "Passive aerodynamic attitude stabilization of near earth satellites. volume I. Librations due to combined aerodynamic and gravitational torques," Tech. rep., DTIC Document, 1961.

[16] Frik, M. A., "Attitude stability of satellites subjected to gravity gradient and aerodynamic torques," AIAA Journal, Vol. 8, No. 10, 1970, pp. 1780-1785.

[17] Shrivastava, S., "Effects of Solar Radiation Pressure and Aerodynamic Forces on Satellite Attitude Dynamics and Their Utilization for Control: A Survey," Journal of the Indian Institute of Science, Vol. 58, No. 9, 1976, p. 391.

[18] Shrivastava, S. and Modi, V., "Satellite attitude dynamics and control in the presence of environmental torques-a brief survey," Journal of Guidance, Control, and Dynamics, Vol. 6, No. 6, 1983, pp. 461-471.

[19] Sarychev, V., "Aerodynamic stabilization of satellites," in "Proc. Inf. Coloquium on Attitude Changes and Stabilization of Satellites," Paris, 1968.

[20] Kumar, R. R., Mazanek, D. D., and Heck, M. L., "Simulation and Shuttle Hitchhiker validation of passive satellite aerostabilization," Journal of spacecraft and rockets, Vol. 32, No. 5, 1995, pp. 806-811.

[21] Kumar, R. R., Mazanek, D. D., and Heck, M. L., "Parametric and classical resonance in passive satellite aerostabilization," Journal of spacecraft and rockets, Vol. 33, No. 2, 1996, pp. 228-234.

[22] Sarychev, V. and Mirer, S., "Relative equilibria of a satellite subjected to gravitational and aerodynamic torques," Celestial Mechanics and Dynamical Astronomy, Vol. 76, No. 1, 2000, pp. 55-68.

[23] Sarychev, V., Mirer, S., Degtyarev, A., and Duarte, E., "Investigation of equilibria of a satellite subjected to gravitational and aerodynamic torques," Celestial Mechanics and Dynamical Astronomy, Vol. 97, No. 4, 2007, pp. 267-287.

[24] Sarychev, V., Mirer, S., and Degtyarev, A., "Equilibria of a satellite subjected to gravitational and 
aerodynamic torques with pressure center in a principal plane of inertia," Celestial Mechanics and Dynamical Astronomy, Vol. 100, No. 4, 2008, pp. 301-318.

[25] Sarychev, V. and Gutnik, S., "Dynamics of an axisymmetric satellite under the action of gravitational and aerodynamic torques," Cosmic Research, Vol. 50, No. 5, 2012, pp. 367-375.

[26] Bak, T. and Wisniewski, R., "Passive Aerodynamic Stabilisation of Low Earth Orbit Satellite," in Spacecraft Guidance, Navigation and Control Systems, Vol. 381, 1997, p. 469.

[27] Chen, Y.-H., Hong, Z.-C., Lin, C.-H., and Chern, J.-S., "Aerodynamic and gravity gradient stabilization for microsatellites," Acta Astronautica, Vol. 46, No. 7, 2000, pp. 491-499.

[28] Psiaki, M. L., "Nanosatellite Attitude Stabilization Using Passive Aerodynamics and Active Magnetic Torquing," in Journal of Guidance, Control, and Dynamics, Vol. 27, 2004, p. 347-355.

[29] Rawashdeh, S. A. and Lumpp, J., "Nano-satellite passive attitude stabilization systems design by orbital environment modeling and simulation," AIAA Infotech.

[30] Maslova, A. and Pirozhenko, A., "Modeling of the aerodynamic moment acting upon a satellite," Cosmic Research, Vol. 48, No. 4, 2010, pp. 362-370.

[31] Sentman, L. H., "Free molecule flow theory and its application to the determination of aerodynamic forces," Tech. rep., Lockheed missiles and space co inc, Sunnyvale CA, 1961.

[32] Schamberg, R., "A New Analytic Representaticn of Surface Interaction with Hypothermal Free Molecule Flow with Application to Neutral-Particle Drag Estimates of Satellites," Tech. Rep. RM-2313, RAND Research Memorandum, 1959.

[33] Cook, G. E., "Satellite drag coefficients," Planetary and Space Science, Vol. 13, No. 10, 1965, pp. 929-946.

[34] Abdel-Jawad, M. M., Goldsworthy, M. J., and Macrossan, M. N., "Stability Analysis of Beagle2 in Free-Molecular and Transition Regimes," Journal of spacecraft and rockets, Vol. 45, No. 6, 2008, pp. 1207-1212.

[35] Santangelo, A. D., Johnson, G. E., "Optimal wing configuration of a Tethered Satellite system in free molecular flow," Journal of spacecraft and rockets, Vol. 29, No. 5, 1992, pp. 668-670.

[36] Wilmoth, R. G., Mitcheltree, R. A., and Moss, J. N., "Low-Density Aerodynamics of the Stardust Sample Return Capsule," Journal of spacecraft and rockets, Vol. 36, No. 3, 1999, pp. 436-441.

[37] Moe, K., Moe, M. M., Levin, D. A., Wysong, I. J., and Garcia, A. L., "Gas-surface interactions in low-Earth orbit," in "AIP Conference Proceedings-American Institute of Physics," Vol. 1333, 2011, p. 1313.

[38] Space engineering: Space environment, ECSS Std. ECSS-E-10-04A, ESA-ESTEC, Noordwijk, The 
Netherlands, 2008.

[39] Ruby, L., "Applications of the Mathieu equation," American Journal of Physics, Vol. 64, No. 1, 1996, pp. 39-44.

[40] Recktenwald, G. D., The stability of parametrically excited systems: coexistence and trigonometrification, Ph.D. thesis, Cornell University, 2006.

[41] Rand, R., Zounes, R., and Hastings, R., "A quasiperiodic Mathieu equation," in "Proceedings of the 1995 Design Engineering Technical Conferences "Vibration of Nonlinear, Random and Time-Varying Systems",", 1995.

[42] Wei, Q., Peers, E., and Huang, X., "Lateral Stability Analysis of Hypersonic Vehicle under Pressure Fluctuation by Solving Mathieu Differential Equation," in "AIAA Atmospheric Flight Mechanics Conference," American Institute of Aeronautics and Astronautics, Reston, Virigina, 2012. 